\title{
HOW MANY ORCHID SPECIES IN COSTA RICA? A REVIEW OF THE LATEST DISCOVERIES
}

\author{
Diego Bogarín
}

Jardín Botánico Lankester, Universidad de Costa Rica, P. O. Box 302-7050, Cartago, Costa Rica; Centro de Investigación en Orquídeas de los Andes “Ángel Andreetta”, Universidad Alfredo Pérez Guerrero, Ecuador diego.bogarin@ucr.ac.or

Abstract. Abstract. Despite its well-established tradition in botanical exploration, which started in 1846 with the visit of Oersted (1846), Costa Rica is still far from having a complete inventory of its orchidaceous flora. After the publication of the most recent and complete treatment of the family by Dressler in 2003, new species and records have been added on a regular basis to the country's inventory. Showy, large-flowered species in previously monographed and botanically well-sampled genera such as Brassia, Dracula, Lycaste, Polycycnis, Stanhopea, and Trichopilia have been described, but the vast majority of species are small-flowered and belong to the subtribes Laeliinae, Pleurothallidinae, and Zygopetalinae. Identifying taxa with ephemeral flowers such as Sobralia is problematic, but a large living collection revealed many new species. Previously described species from other countries have regularly been recorded in Costa Rica. These new records have floristic affinities mainly with the floras of Panama, Colombia, Ecuador, and Venezuela. As an example, Acianthera aberrans, Epidendrum scharfii, Epidendrum stellidifforme, Lockhartia chocoensis, Maxillaria bolivarensis, Ornithidium pendulum, Ornithocephalus montealegrae, and Warmingia zamorana have been found in both Costa Rica and Ecuador. The genus Uleiorchis with the Venezuelan species Uleiorchis ulaei, identified in the MO herbarium by Ron Liesner, constitutes an interesting new record in Costa Rica. Maxillaria appendiculoides, first described from Costa Rica, has recently been reported from Ecuador. Campylocentrum tenellum, Lepanthes droseroides, Lepanthes mariposa, and Sobralia bouchei from Panama were also lately collected in Costa Rica. Although much floristic work remains to be completed and the country has significant areas that are poorly sampled, the establishment of large and documented collections of living plants at Lankester Botanical Garden, associated with an increasing access to critical documentation (types and literature), have been the keys to improving our understanding of orchid diversity in Costa Rica and its floristic relationships with other areas.

Resumen. A pesar de su bien establecida tradición en exploración botánica, la cual comenzó en 1846 con la visita de Oersted (1846), Costa Rica está todavía lejos de tener un inventario completo de su flora de orquídeas. Después de la publicación del tratamiento más reciente y completo de la familia por Dressler (2003) nuevas especies y registros han sido añadidos regularmente al inventario del país. Especies llamativas, de flores grandes han sido descritas en géneros previamente monografiados y botánicamente bien muestreados, tales como: Brassia, Dracula, Lycaste, Polycycnis, Stanhopea, y Trichopilia, pero la gran mayoría de especies son plantas de flores pequeñas que pertenecen a las subtribus Laeliinae, Pleurothallidinae, y Zygopetalinae. Las flores efímeras, como Sobralia tienen problemas especiales con su identificación pero una colección viva, grande, revela muchas nuevas especies. Las especies previemente descritas de otros países han sido registradas en Costa Rica con regularidad. Estos nuevos registros tienen afinidades florísticas principalmente con la flora de Panamá, Colombia, Ecuador, y Venezuela. Por ejemplo, Acianthera aberrans, Epidendrum scharfii, Epidendrum stellidifforme, Lockhartia chocoensis, Maxillaria bolivarensis, Ornithidium pendulum, Ornithocephalus montealegrae, y Warmingia zamorana han sido encontradas tanto en Costa Rica como en Ecuador. El género Uleiorchis con la especie venezolana Uleiorchis liesneri, identificado en el herbario MO por Ron Liesner, constituye un nuevo registro interesante en Costa Rica. También, Maxillaria appendiculoides descrita de Costa Rica, ha sido registrada recientemente en Ecuador. Campylocentrum tenellum, Lepanthes droseroides, Lepanthes mariposa, y Sobralia bouchei de Panamá también fueron colectadas recientemente en Costa Rica. Aunque mucho trabajo florístico queda por ser completado y el país tiene áreas significativas que 
están mal muestreadas, el establecimiento de colecciones grandes y documentadas de plantas vivas en el Jardín Botánico Lankester, asociado con un acceso creciente a la documentación crítica (tipos y literatura), han sido la llave para el mejoramiento de nuestro entendimiento de la diversidad de orquídeas en Costa Rica y sus relaciones florísticas con otras áreas.

KEY wORDS: Orchidaceae, Costa Rica, novelties

Despite its well-established tradition in botanical exploration, which started in 1846 with the visit of Oersted (1846), Costa Rica is still far from having a complete inventory of its orchidaceous flora (Bogarín et al., 2008). During the second half of the 19th century Hemsley (1883) and Reichenbach (1886) published the first inventories for the orchids of Mesoamerica. Later, Schlechter (1918), Ames (in Standley, 1937), and Williams (1956) updated the species' list for Central America. The need for an up-to-date checklist of the Orchidaceae in Costa Rica was filled in part by Mora-Retana and García (1992), Pupulin (2002), and Dressler (1993; 2003). More recently, Ossenbach et al. (2007) published a checklist of the orchids of Costa Rica and Mesoamerica.

Throughout the history of documentation of the Orchidaceae in Costa Rica, more than three quarters of the species were named by botanists who deposited the material outside the country. Although botanical exploration began almost 150 years ago, the documentation of the orchid flora was developed sporadically by botanists during the past 15 decades. In the 19th century, Bateman, Lindley, Reichenbach, and Rolfe named the first species known for the country. Orchid research declined from 1860 to 1900 until Ames and Schlechter worked intensively on the Neotropical Orchidaceae (Fig. 1). However, the number of species named declined dramatically between 1950 and 1960 , when less than 30 species were described for Costa Rica, contrasting with the decade of 1920 when nearly 300 of the species were named (Fig. 1). Even though there was botanical exploration in those years, the botanists never managed to document permanently the flora of Costa Rica. This was mainly attributable to the lack of critical materials to identify the species and also the fact that the literature was often published as a few copies that circulated only in developed countries.

The Global Taxonomy Initiative (GTI) of the Convention on Biological Diversity (CBD) pointed out a critical problem for effective conservation management that largely depends on taxonomic knowledge: "the inadequate taxonomic information and infrastructure, coupled with declining taxonomic expertise." Unfortunately, one of the main problems developing taxonomic expertise in tropical countries has been the lack of adequate information for species identification. The existence of this taxonomic impediment is the major problem to solve by global strategies such as GTI. With the establishment of Lankester Botanical Garden (LBG) in 1973 and other institutions such as Museo Nacional de Costa Rica and Instituto Nacional de Biodiversidad (INBio) there has been an increase in orchid research that has continued until the present. It has been demonstrated that LBG has played a major role in revealing orchid diversity through a well-developed taxonomic system (Fig. 1). The mission of LBG has been to eliminate the taxonomic impediment through gathering critical information and making it available for researchers and students.

Floristics and botanical exploration in Costa Rica are the basis for future research and conservation actions. New techniques for species identification using DNA sequences or DNA barcoding have been developed; however, a well-established taxonomic system is needed to solve the problem of incomplete floristic inventories and for developing new techniques that

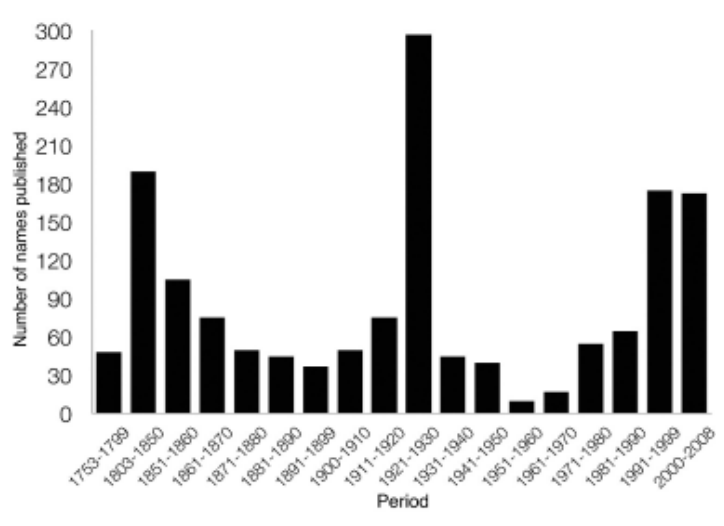

FIgURE 1. Comparison of the number of species of orchids described from Costa Rica at different periods. 
will not work without an adequate taxonomic basis (Lahaye et al., 2008). The aim of the present paper is to discuss the status of the Orchidaceae in Costa Rica, where new species are continuously being discovered.

\section{A review of the latest discoveries}

After the publication of the most complete treatment of the Orchidaceae from Costa Rica by Dressler (2003), new species and new records have been added on a regular basis to the country's orchid checklist. Ossenbach et al. (2007) estimated that 1461 orchid species occur in Costa Rica. After Ossenbach et al. (2007), 34 species were described as new to science and 24 species previously described outside Costa Rica were recorded for this country. The number now stands at 1519 species. A summary of the latest discoveries and a list of additional material of several species known from a single locality or specimen are provided in Table 1 with synonyms, authorities, and voucher information. Endemic species are annotated there with an asterisk.

Several species recorded by Ossenbach et al. (2007) are listed here with their respective voucher. Among the species cited, 29 are endemic to Costa Rica. There are 25 species found both in Costa Rica and Panama, and 14 shared with Ecuador. Some disjunctions are recorded, such as Epidendrum stellidifforme and Warmingia zamorana, which are known only from Costa Rica and Ecuador, but their presence in Panama and Colombia is highly probable (Bogarín et al., 2008). Also, there are 16 species widespread from Costa Rica to South America and 10 ranging from Costa Rica to Nicaragua, Honduras, Guatemala or Mexico.

Subtribe Angraecinae: Campylocentrum tenellum Todzia is recorded for a first time in Costa Rica. Two specimens of $C$. tenellum from different localities in Costa Rica are known, but unfortunately in sterile condition. A recent fertile collection provided a proper recording of this species (Fig. 2a, 3c). An aphyllous species with flat roots was recorded from El Valle del General on the Pacific watershed of the Cordillera de Talamanca. Although C. pachyrrhizum (Rchb.f.) Rolfe is expected to occur in Costa Rica, the material collected indicates that it may correspond to an undescribed species. Campylocentrum multiflorum Schltr. is accepted and considered as distinct from $C$. fasciola (Lindl.) Cogn. The name C. tyrridion Garay \& Dunst. should be best applied to the species from Venezuela. The Costa Rican voucher of $C$. dressleri H.Dietr. \& M.A.Díaz (J. Villalobos s.n. (USJ!)) clearly corresponds to $C$. multiflorum Schltr., so $C$. dressleri is excluded from Costa Rica (Bogarín and Pupulin, 2009).

Tribe Gastrodieae: An interesting record of the holomycotrophic orchid genus Uleiorchis is U. ulaei (Cogn.) Handro, which was recently identified by Ron Liesner at MO. The species had been recorded in Honduras, Panama, and South America.

Subtribe Goodyerinae: Studies published by Ormerod (2007, 2008) revealed a new species of Aspidogyne, A. grayumii Ormerod, known from the Estación Biológica Las Cruces in southern Costa Rica near Panama. A new Kreodanthus, K. curvatus Ormerod, is known from the northwest slope of Volcán Barva in the Central Cordillera and also from a collection in the Cordillera de Talamanca. It is compared with the Panamanian $K$. bugabae Ormerod. Two new species of Microchilus were also recorded $-M$. maasii Ormerod from the Monteverde region (also from central Panama) and M. tessellatus Ormerod, endemic to Costa Rica. The recently described $M$. valverdei Ormerod is now a synonym of M. calophyllus (Rchb.f.) Ormerod. Also, Platythelys alajuelae Ormerod is endemic to Costa Rica and known from the Cordillera de Tilarán and the Cordillera Central.

Subtribe Laeliinae: Preliminary results of a revision of Encyclia in Costa Rica yielded a new record: Encyclia gravida (Lindl.) Schltr. Plants of this species are usually recognized by having cleistogamous flowers. The number of new species and records is considerable in the large genus Epidendrum. The additions have been mainly published by Hágsater and co-workers $(2004,2006,2008)$. The following new species have been described: E. apatotylosum Hágsater, E. astro-selaginellum Hágsater \& E.Santiago, E. bicuniculatum Hágsater \& E.Santiago, E. isthmoides Hágsater \& E.Santiago (recorded recently; Fig. 6d), E. xnocteburneum Hágsater \& L.Sánchez (a natural hybrid between E. eburneum Rchb.f. and E. nocturnum Jacq.), E. philowercklei Hágsater \& E.Santiago, E. salpichlamys Hágsater \& E.Santiago, E. steno- 
TABLE 1. List of recent additions to the orchid flora of Costa Rica

Species

1. Acianthera aberrans (Luer) Pupulin \& Bogarín, Lankesteriana 8(2): 53-55. 2008.

2. Acianthera cabiriae Pupulin, G.A.Rojas \& J.D.Zuñiga, Harvard Pap. Bot. 12(1): 160. 2007.*

3. Acianthera fecunda Pupulin, G.A.Rojas \& J.D.Zuñiga, Harvard Pap. Bot. 12(1): 158. 2007. *

4. Acianthera hamata Pupulin \& G.A.Rojas, Harvard Pap. Bot. 12(1): 156. 2007.*

5. Aspidogyne grayumii Ormerod, Harvard Pap. Bot. 11(2): 147. 2007. *

6. Barbosella orbicularis Luer, Selbyana 3(1,2): 10-11, f. 108. 1976.

7. 'Bensteinia ramonensis Pupulin, Selbyana 28(2): 113. 2008. *

8. Campylocentrum tenellum Todzia, Ann. Missouri Bot. Gard. 72: 877. 1985.

9. Dichaea gomez-lauritoi Pupulin, Harvard Pap. Bot. 12(1): 87. 2007. *

10. Dryadella fuchsii Luer, Monogr. Syst. Bot. Missouri Bot. Gard.76: 162-163. 1999.

11. Echinosepala lappiformis (A.H.Heller \& L.O.Williams) Pridgeon \& M.W.Chase Lindleyana 17(2): 101. 2002. Pleurothallis lappiformis

A.H.Heller \& L.O.Williams, Fieldiana, Bot. 31(2): 42, f. 8. 1964. Myoxanthus lappiformis (A.H.Heller \& L.O.Williams) Luer, Monogr. Syst. Bot. Missouri Bot. Gard. 15: 38. 1986. Brenesia lappiformis (A.Heller \& L.O.Williams) Luer, Monogr. Syst. Bot. Missouri Bot. Gard.95: 255. 2004. Echinella lappiformis (A.Heller \& L.O.Williams) Pridgeon \& M.W.Chase, Lindleyana 16(4): 253. 2001.

12. Elleanthus ligularis Dressler \& Bogarín, Lankesteriana 7(3): 539. 2007.

13. Encyclia gravida (Lindl.) Schltr., Beih. Bot. Centralbl.36(2): 472.1918. Epidendrum gravidum Lindl., J. Hort. Soc. London 4: 114. 1849.

14. Epidendrum acroamparoanum Hágsater \& L.Sánchez S., Icon. Orchid. (Mexico) 8: t. 802. 2006. *

15. Epidendrum atypicum Hágsater \& E. Santiago A., Icon. Orchid. (Mexico) 8: t. 810. 2006. *

16. Epidendrum angustilobum Fawc. \& Rendle, J. Bot. 47(556): 124-125. 1909. Epidendrum latifolium (Lindl.) Garay \& H.R. Sweet, J. Arnold Arbor 53(3): 392. 1972.

17. Epidendrum apatotylosum Hágsater, Icon. Orchid. 9: t. 909. 2007. *

18. Epidendrum arcuiflorum Ames \& C.Schweinf., Schedul. Orch. 10: 58-59. 1930. Epidendrum powellii Schltr., Repert. Spec. Nov. Regni Veg. Beih. 17: 38. 1922.

19. Epidendrum astroselaginellum Hágsater \& E.Santiago, Icon. Orchid. 9: t. 910. 2007.

20. Epidendrum bicuniculatum Hágsater \& E.Santiago, Icon. Orchid. 9: t. 913. 2007. *

21. Epidendrum buenaventurae F.Lehm. \& Kränzl., Bot. Jahrb. Syst. 26(5): 470-471. 1899

22. Epidendrum chalcochromum Hágsater, Icon. Orchid. (Mexico) 8: t. 818. 2006.*
Voucher

Pupulin 4857, JBL

Karremans 1433, CR

Bogarín 2650, CR

Quesada Chanto s.n., CR

Grayum 9267, MO

Bogarín 1949, JBL

Bogarín 1923, CR

Bogarín 5844, JBL

Gómez-Laurito 8174, CR

Standley \& Valerio 45606, AMES

Dressler 6768, JBL

Dressler 6836B, CR

Pupulin 5377, JBL

Morales 4737, MO

Weber 6081, AMES

Moraga 312, INB

Horich sub E. Hágsater 7117, INB

Herrera 3595, CR

Gómez 23814, MO

Grayum \& G. Herrera 7716, MO

Gómez 19984, MO

Hágsater \& Mora de Retana 9495 , INB 


\begin{tabular}{|c|c|}
\hline Species & Voucher \\
\hline $\begin{array}{l}\text { 23. Epidendrum cristatum Ruiz \& Pav., Syst. Veg. Fl. Peruv. Chil. 1: } 243 . \\
\text { 1798. Epidendrum raniferum Lindl., Gen. Sp. Orchid. P1.109. 1831. }\end{array}$ & Brade 1281, AMES \\
\hline $\begin{array}{l}\text { 24. Epidendrum dolichochlamys Hágsater \& E.Santiago A., Icon. Orchid. } \\
\text { (Mexico) 8: t. 829. 2006. * }\end{array}$ & Chavarría $661, \mathrm{INB}$ \\
\hline $\begin{array}{l}\text { 25. Epidendrum gibbosum L.O.Williams, Ann. Missouri Bot. Gard. 28(4): 420- } \\
\text { 421, pl. 21, 7-10. 1941. Epidendrum acrostigma Hágsater \& García-Cruz, } \\
\text { Icon. Orchid. 3: t. 301. 1999. }\end{array}$ & Grayum 4578, CR \\
\hline $\begin{array}{l}\text { 26. Epidendrum haberi Hágsater \& L.Sánchez S., Icon. Orchid. (Mexico) 8: t. } \\
\text { 840. 2006.* }\end{array}$ & Haber \& Cruz 7245, INB \\
\hline 27. Epidendrum hawkesii A.H.Heller, Phytologia 14(1): 2-4, t. 1. 1966. & Horich sub Hágsater 7649, AMO \\
\hline $\begin{array}{l}\text { 28. Epidendrum isthmoides Hágsater \& E.Santiago, Icon. Orchid. 11: t. } 1133 . \\
\text { 2008. * }\end{array}$ & Bello $1516, \mathrm{INB}$ \\
\hline $\begin{array}{l}\text { 29. Epidendrum maduroi Hágsater \& García-Cruz, Icon. Orchid. (Mexico) 3: } \\
\text { pl. 352. } 1999 .\end{array}$ & Bogarin 1465, JBL \\
\hline $\begin{array}{l}\text { 30. Epidendrum microrigidiflorum Hágsater, Icon. Orchid. (Mexico) 8: t. } 853 . \\
2006 .\end{array}$ & Dressler 6259, AMO \\
\hline $\begin{array}{l}\text { 31. Epidendrum 'nocteburneum Hágsater \& L.Sánchez, Icon. Orchid. 11: t. } \\
\text { 1148. } 2008 .\end{array}$ & $\begin{array}{l}\text { Weston } 57 \text { sub Rodríguez 1042, } \\
\text { USJ }\end{array}$ \\
\hline $\begin{array}{l}\text { 32. Epidendrum orthodontum Hágsater \& L.Sánchez, Icon. Orchid. 3: t. } 361 . \\
1999 .\end{array}$ & Karremans 2255, JBL \\
\hline $\begin{array}{l}\text { 33. Epidendrum pachytepalum Hágsater \& E.Santiago A., Icon. Orchid. } \\
\text { (Mexico) 8: t. 865. } 2006 .\end{array}$ & Burger $8253, \mathrm{~F}$ \\
\hline $\begin{array}{l}\text { 34. Epidendrum pendens L.O.Williams, Ann. Missouri Bot. Gard. 28(4): 421- } \\
\text { 422, pl. 23. } 1941 .\end{array}$ & Haber \& Cruz 7986, CR \\
\hline $\begin{array}{l}\text { 35. Epidendrum philowercklei Hágsater \& E.Santiago A., Icon. Orchid. } \\
\text { (Mexico) 8: t. 870. } 2006 .\end{array}$ & Fernández 677, CR \\
\hline $\begin{array}{l}\text { 36. Epidendrum platystomoides Hágsater \& L.Sánchez S., Icon. Orchid. } \\
\text { (Mexico) 8: t. 872. 2006. * }\end{array}$ & $\begin{array}{l}\text { Hágsater \& Mora de Retana 9507, } \\
\text { AMO }\end{array}$ \\
\hline $\begin{array}{l}\text { 37. Epidendrum purpurascens H.Focke, Tijdschr. Wis- Natuurk. Wetensch. } \\
\text { Eerste K1. Kon. Ned. Inst. Wetensch.) 4: 64-65. 1851. Epidendrum } \\
\text { glumibracteum Rchb.f., Hamburger Garten- Blumenzeitung 19: 11. } 1863 .\end{array}$ & Valerio 1017, CR \\
\hline $\begin{array}{l}\text { 38. Epidendrum rousseauae Schltr., Beih. Bot. Centralbl., Abt. } 2 \text { 36(2): 407- } \\
\text { 408. } 1918 .\end{array}$ & Todzia 552, CR \\
\hline $\begin{array}{l}\text { 39. Epidendrum salpichlamys Hágsater \& E.Santiago A, Icon. Orchid. } \\
\text { (Mexico) 8: t. 883. 2006. * }\end{array}$ & Grayum \& Pam Sleeper 3446, MO \\
\hline 40. Epidendrum scharfii Hágsater \& Dodson, Icon. Orchid. 2: t. 185. 1993. & Pupulin 6500, JBL \\
\hline $\begin{array}{l}\text { 41. Epidendrum serruliferum Schltr., Repert. Spec. Nov. Regni Veg. Beih. 19: } \\
\text { 44. 1923. * }\end{array}$ & $\begin{array}{l}\text { Wercklé } 114, \mathrm{~B} \text {, destroyed; drawing } \\
\text { of type, AMES }\end{array}$ \\
\hline $\begin{array}{l}\text { 42. Epidendrum stellidifforme Hágsater \& Dodson, Icon. Orchid. (Mexico) 4: } \\
\text { t. } 487.2001 .\end{array}$ & Bogarín 2814, JBL \\
\hline $\begin{array}{l}\text { 43. Epidendrum stenoselaginellum Hágsater \& E.Santiago, Icon. Orchid. 9: t. } \\
\text { 991. } 2007 .\end{array}$ & Hágsater 11115, AMO \\
\hline $\begin{array}{l}\text { 44. Epidendrum tritropianthum Hágsater \& E.Santiago A., Icon. Orchid. } \\
\text { (Mexico) 9(6): t. 998. } 2007 .\end{array}$ & Ingram \& Ferrell 743, INB \\
\hline 45. Epidendrum villotae Hágsater \& Dodson, Icon. Orchid. 3: pl. 398. 1999. & Herrera 5151, MO \\
\hline
\end{tabular}




\begin{tabular}{|c|c|}
\hline Species & Voucher \\
\hline 46. Epidendrum vulcanicola A.H.Heller, Fieldiana, Bot. 32(2): 11, f. 2.1968. & Alcázar 56, USJ \\
\hline $\begin{array}{l}\text { 47. Epidendrum vulgoamparoanum Hágsater \& L.Sánchez S., Icon. Orchid. } \\
\text { (Mexico) 8: t. 898. } 2006 .\end{array}$ & Hágsater 6963, INB \\
\hline $\begin{array}{l}\text { 48. Epidendrum zunigae Hágsater, Karremans \& Bogarín, Lankesteriana 8(2): } \\
\text { 63. 2008. * }\end{array}$ & Bogarin 2680, JBL \\
\hline $\begin{array}{l}\text { 49. Gongora boracayanensis Jenny, Dalström \& W.E.Higgins, Selbyana 28(2): } \\
\text { 99. 2007. * }\end{array}$ & Clark et al. 314 B, SEL \\
\hline $\begin{array}{l}\text { 50. Kefersteinia alata Pupulin, Harvard Pap. Bot. 8(2): 161-164, f. 1, 2A-B. } \\
2004 .\end{array}$ & Carman s.n,, JBL \\
\hline 51. Kefersteinia saccata Pupulin, Willdenowia 38(1): 188. 2008. * & Pupulin 6549, CR \\
\hline 52. Kreodanthus curvatus Ormerod, Harvard Pap. Bot. 13(1): 61. 2008. * & Grayum 7481 , MO \\
\hline 53. Lepanthes droseroides Luer, Lindleyana 2: 188. 1987. & Bogarin 5234, JBL \\
\hline $\begin{array}{l}\text { 54. Lepanthes gratiosa Pupulin \& D.Jiménez, Orchid Digest 73: 137-139. } \\
\text { 2009. * }\end{array}$ & Pupulin $7350, \mathrm{CR}$ \\
\hline $\begin{array}{l}\text { 55. Lepanthes machogaffensis Pupulin \& D. Jiménez, Orchid Digest 73: 139- } \\
\text { 140. 2009. * }\end{array}$ & Pupulin $7308, \mathrm{CR}$ \\
\hline 56. Lepanthes mariposa Luer, Phytologia 55: 187.1984. & Bogarin 5034, JBL \\
\hline 57. Lepanthes pelvis Pupulin \& D.Jiménez, Orchid Digest 73: 142-144. 2009. * & Pupulin $7336, \mathrm{CR}$ \\
\hline $\begin{array}{l}\text { 58. Lockhartia chocöensis Kränzl. in H.G.A.Engler (ed.), Pflanzenr., IV, } \\
\text { 50(83): 19. } 1923 .\end{array}$ & Bogarin 2352, JBL \\
\hline 59. Lycaste angelae Oakeley, Lycaste, Ida, Anguloa 27. 2008. & Oakeley A5 (CR7), K-HFO \\
\hline $\begin{array}{l}\text { 60. Lycaste bruncana Bogarín, Lankesteriana 7(3): 543. 2007. Lycaste } \\
\text { crystallina Wubben ex Oakeley, Lycaste, Ida, Anguloa 73. 2008. * }\end{array}$ & Bogarin 3987, CR \\
\hline 61. Lycaste x daniloi Oakeley, Lycaste, Ida, Anguloa 76. 2008. & Oakeley 14 (CR14), K-HFO \\
\hline $\begin{array}{l}\text { 62. Lycaste panamanensis (Fowlie) Oakeley, Orchid Digest 71: 205. } 2007 . \\
\text { Lycaste macrophylla subsp. panamanensis Fowlie, Lasca Leaves 14(1): 40, } \\
\text { f. } 1964 .\end{array}$ & see Oakeley (2008) \\
\hline 63. Maxillaria bolivarensis C.Schweinf., Bot. Mus. Leafl. 20: 22. 1962. & Whitten 2030, JBL \\
\hline 64. Microchilus maasii Ormerod, Harvard Pap. Bot. 11(2): 161. 2007. & Bello 2809, CR \\
\hline 65. Microchilus tessellatus Ormerod, Harvard Pap. Bot. 11(2): 172. 2007. * & Grayum 8925, MO \\
\hline 66. Ornithidium pendulum (Poepp. \& Endl.) Cogn., Fl. Bras.3(6): 92. 1904. & Karremans 448, JBL \\
\hline 67. Palmorchis nitida Dressler, Die Orchidee 34(1): 29-30. 1983. & Gómez 26248, USJ \\
\hline 68. Platythelys alajuelae Ormerod, Harvard Pap. Bot. 11(2): 174. 2007. * & Ingram \& Ferrell 680, MO \\
\hline $\begin{array}{l}\text { 69. Pleurothallis bogarinii Pupulin \& J.D.Zuñiga, Orchids } 76 \text { (Lindleyana): } \\
\text { 690. 2007.* }\end{array}$ & Pupulin 5285, CR \\
\hline $\begin{array}{l}\text { 70. Pleurothallis caudatipetala C.Schweinf., Bot. Mus. Leafl.10: 175, t. } \\
\text { 22, f. 5-8. 1942. Specklinia caudatipetala (C. Schweinf.) Luer, Monogr. } \\
\text { Syst. Bot. Missouri Bot. Gard.95: 259. 2004. Panmorphia caudatipetala } \\
\text { (C. Schweinf.) Luer, Monogr. Syst. Bot. Missouri Bot. Gard.105: } 150 . \\
\text { 2006. }\end{array}$ & Luer 12137, MO \\
\hline
\end{tabular}

71. Pleurothallis duplooyi Luer \& Sayers, Revista Soc. Boliv. Bot.3(1/2): 4850. 2001. Specklinia duplooyi (Luer \& Sayers) Luer, Monogr. Syst. Bot. Missouri Bot. Gard. 95: 260. 2004. Panmorphia duplooyi (Luer \& Sayers) Luer, Monogr. Syst. Bot. Missouri Bot. Gard. 105: 153. 2006. 


\begin{tabular}{|c|c|}
\hline Species & Voucher \\
\hline $\begin{array}{l}\text { 72. Pleurothallis minutalis Lindl., Fol. Orchid. Pleurothallis 40. 1859. Humbol- } \\
\text { tia minutalis (Lindl.) Kuntze, Revis. Gen. P1.2: 668. 1891. Humboldtia } \\
\text { minutalis (Lindl.) Kuntze, Revis. Gen. P1.2: 668. 1891. Panmorphia } \\
\text { minutalis (Lind1.) Luer, Monogr. Syst. Bot. Missouri Bot. Gard.105: } 167 . \\
\text { 2006. Specklinia minutalis (Lindl.) Luer, Monogr. Syst. Bot. Missouri Bot. } \\
\text { Gard. 95: 262. 2004. Anathallis minutalis (Lindl.) Pridgeon \& M.W. Chase, } \\
\text { Lindleyana 16(4): 249. 2001. }\end{array}$ & Endrés s.n., W \\
\hline 73. Restrepiella lueri Pupulin \& Bogarín, Willdenowia 37(1): 325. 2007. * & Bogarín 3009, CR \\
\hline 74. Scaphyglottis robusta B.R.Adams, Phytologia 64: 253. 1988. & Bogarin 2662, JBL \\
\hline 75. Sobralia aspera Dressler \& Pupulin, Orquideología 25(2): 148. 2008. * & Dressler 6783, CR \\
\hline 76. Sobralia blancoi Dressler \& Pupulin, Orquideología 25(2): 139. 2008. * & Dressler 6706, CR \\
\hline 77. Sobralia bouchei Ames \& C.Schweinf., Schedul. Orchid. 10: 4. 1930. & Bogarin 4201, JBL \\
\hline 78. Sobralia pendula Dressler \& Pupulin, Orquideología 25(2): 140. 2008. * & Dressler 6746, CR \\
\hline 79. Sobralia rarae-avis Dressler, Orquideología 25(1): 38. 2007. * & Dressler 6793, CR \\
\hline 80. Trichopilia primulina Dressler \& Bogarín, Orchideen J. 16(2): 60. 2009. * & Dressler 7030, CR \\
\hline $\begin{array}{l}\text { 81. Uleiorchis ulaei (Cogn.) Handro, Arq. Bot. Estado São Paulo 3(4): } 175 . \\
\text { 1958. Wullschlaegelia ulaei Cogn., Fl. Bras. 3(4): 244. 1895. }\end{array}$ & Hammel 11657, MO \\
\hline $\begin{array}{l}\text { 82. Warmingia zamorana Dodson, Icon. P1. Trop., II, 6: t. 599. } 1989 . \\
\text { Warmingia margaritacea B.Johans., Lindleyana 7: 194. } 1992 .\end{array}$ & Karremans 1123, JBL \\
\hline
\end{tabular}

selaginellum Hágsater \& E.Santiago (from Nicaragua, Costa Rica, and Panama), and E. zunigae Hágsater, Karremans \& Bogarín (Fig. 4g, 5d). The following new species were described by Hágsater et al. (2006) and recorded by Ossenbach et al. (2007): Epidendrum acroamparoanum Hágsater \& L.Sánchez, E. atypicum Hágsater \& E.Santiago, E. chalcochromum Hágsater, E. dolicho-chlamys Hágsater \& E.Santiago, E. haberi Hágsater \& L.Sánchez, E. microrigidiflorum Hágsater, E. pachytepalum Hágsater \& E. Santiago, E. philowercklei Hágsater \& E. Santiago, E. platystomoides Hágsater \& L.Sánchez, E. salpichlamys Hágsater \& E.Santiago, E. tritropianthum Hágsater \& E.Santiago, and E. vulgoamparoanum Hágsater \& L.Sánchez (previously known as E. amparoanum Schltr., which is now treated as a synonym of E. barbeyanum Kränzl.). Epidendrum microrigidiflorum, E. pachytepalum, E. philowercklei, and E. vulgoamparoanum are found in both Costa Rica and Panama. New collections of previously described species have been added regularly: E. buenaventurae F.Lehm. \& Kränzl., E. scharfii Hágsater \& Dodson (Fig. 4f), E. stellidifforme Hágsater \& Dodson (Fig. 4e, 5c), and E. villotae Hágsater \& Dodson, all recorded previously from
Ecuador; Epidendrum hawkesii A.H.Heller and E. vulcanicola A.H.Heller from Nicaragua; and E. maduroi Hágsater \& García-Cruz (Fig. 4d), Epidendrum orthodontum Hágsater \& L.Sánchez (Fig. 5b), E. pendens L.O.Williams, and E. rousseauae Schltr. from Panama, which was formerly listed by Pupulin (2002). The following taxonomic changes affecting Costa Rican species have been noted by Hágsater (2008): Epidendrum angustilobum Fawc. \& Rendle (=E. latifolium (Lindl.) Garay \& H.R.Sweet), E. arcuiflorum Ames \& C.Schweinf. (=E. powellii Schltr.), E. cristatum Ruiz \& Pav. (=E. raniferum Lind1.), E. gibbosum L.O.Williams (=E. acrostigma Hágsater \& García-Cruz), E. purpurascens H.Focke (=E. glumibracteum Rchb.f.), and the reconsidered $E$. serruliferum Schltr. Two new species and a new addition will be soon published by Pupulin and Karremans (unpublished). A collection of Scaphyglottis robusta B.R.Adams, previously described from Panama, has been documented in tropical wet premontane forest at an elevation of $1150 \mathrm{~m}$ in the Caribbean watershed of the Cordillera de Talamanca (Fig. 10b). Another specimen was collected in the region of Turrialba in the province of Cartago (Fig. $4 \mathrm{j}$ ). 

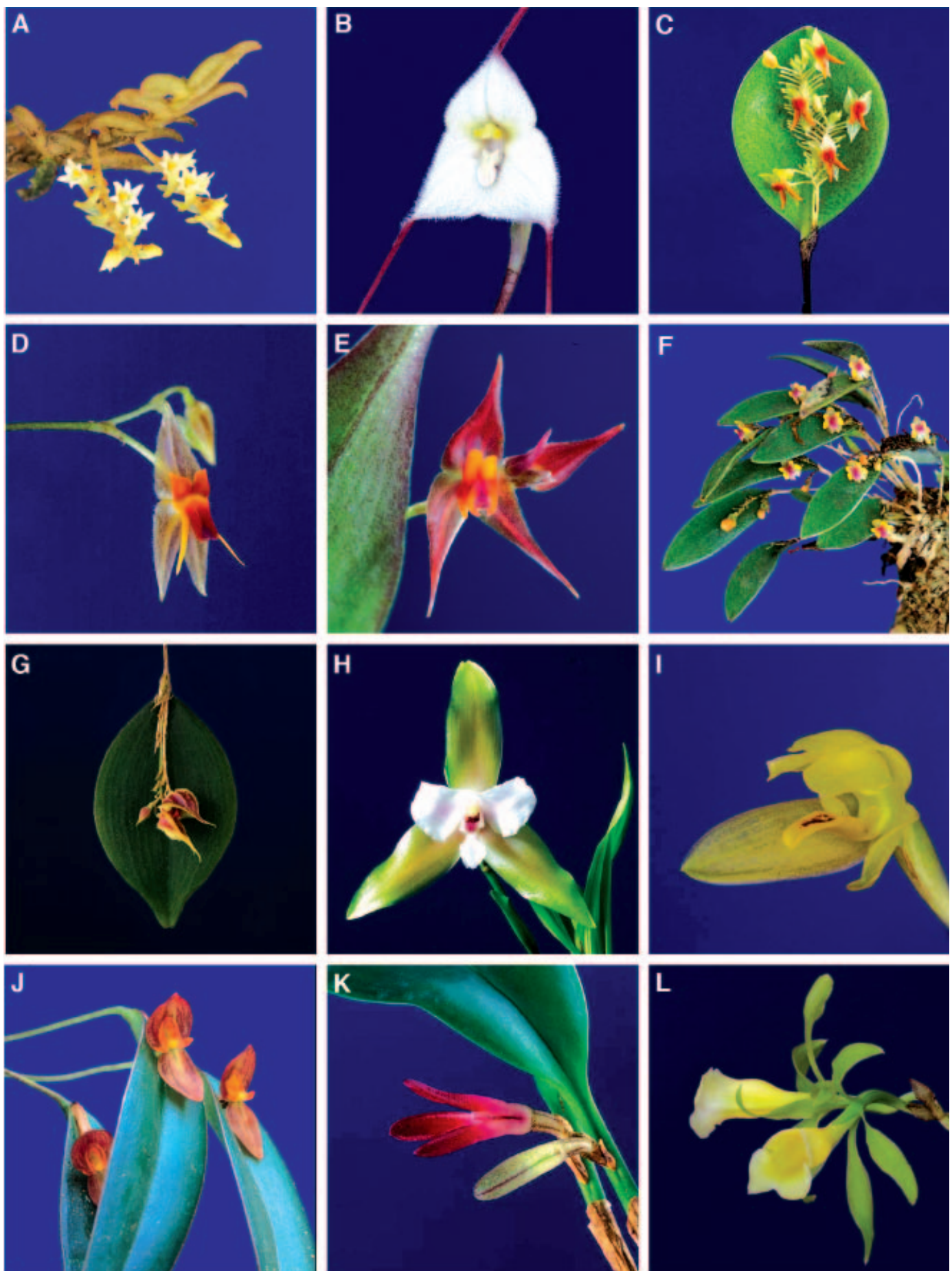

FIGURE 2. Photographs of: A. Campylocentrum tenellum; B. Dracula maduroi; C. Lepanthes droseroides; D. Lepanthes gratiosa; E. Lepanthes machogaffensis; F. Lepanthes mariposa; G. Lepanthes pelvis; H. Lycaste bruncana; I. Mormolyca fumea; J. Pleurothallis bogarinii; K. Restrepiella lueri; L. Trichopilia primulina. Photographs: A-C, F, I, K, L by Diego Bogarín; D, E, G by Franco Pupulin. 


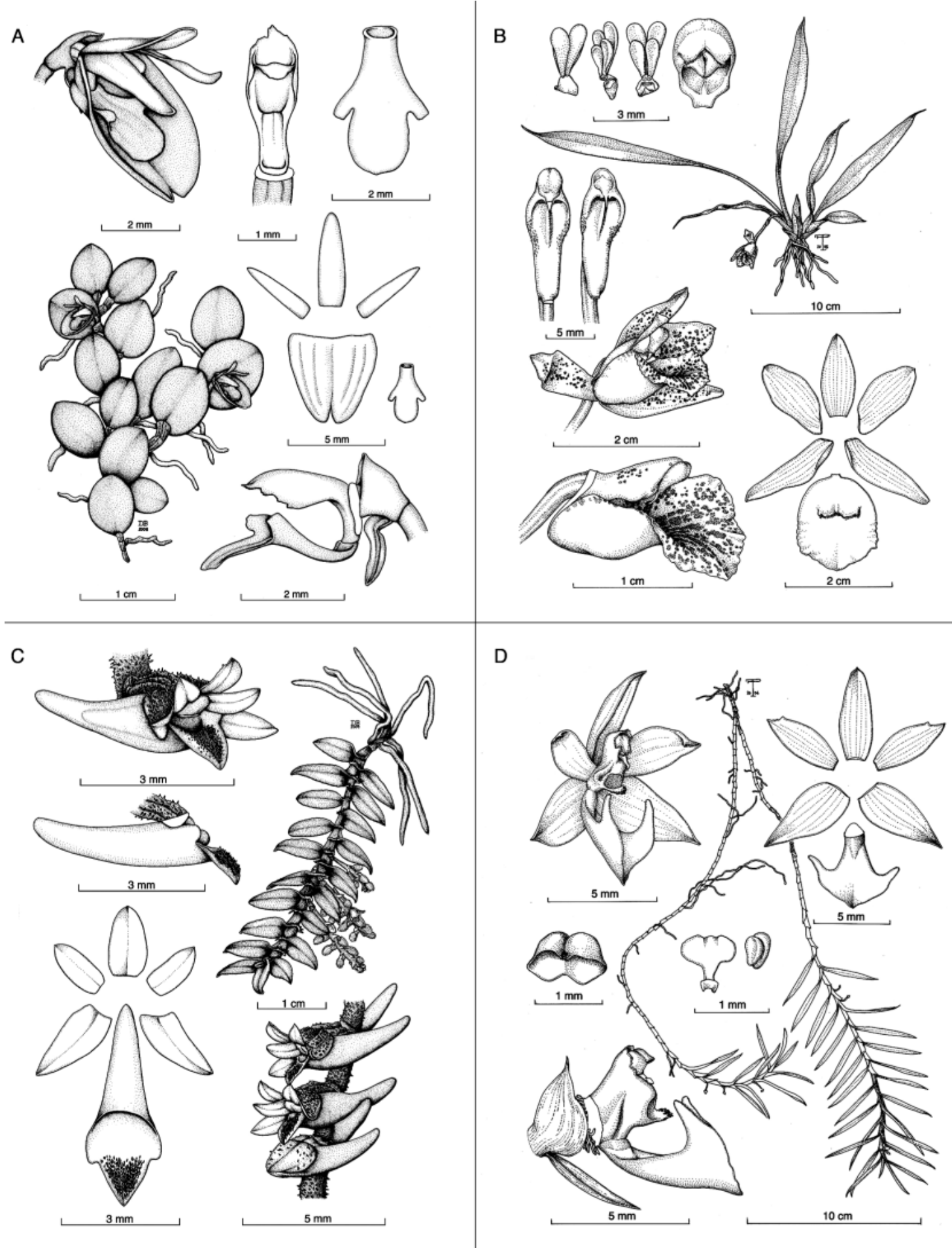

FiguRE 3. Drawings of: A. Barbosella orbicularis; B. × Bensteinia ramonensis; C. Campylocentrum tenellum; D. Dichaea gomez-lauritoi. Drawings: A, C by Diego Bogarín; B, D by Franco Pupulin. 

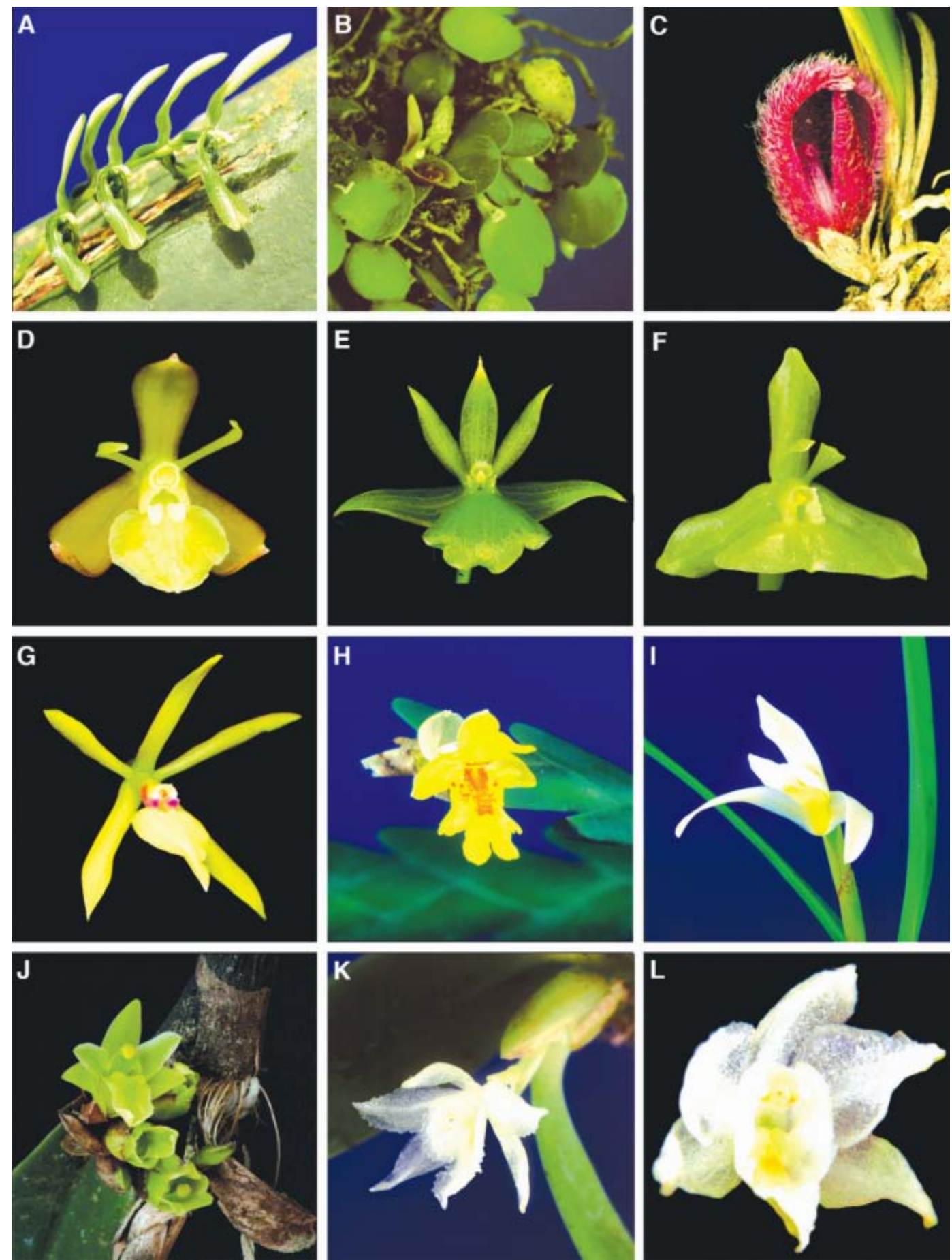

FIGURE 4. Photographs of: A. Acianthera aberrans; B. Barbosella orbicularis; C. Brenesia lappiformis; D. Epidendrum maduroi; E. Epidendrum scharfii; F. Epidendrum stellidifforme; G. Epidendrum zunigae; H. Lockhartia chocoensis; I. Maxillaria bolivarensis; J. Scaphyglottis robusta; K. Warmingia zamorana (Costa Rica); L. Warmingia zamorana (Ecuador); Photographs A, H, K by Franco Pupulin; B, I, J by Diego Bogarín; C-F courtesy of LBG; L courtesy of CIOA. 


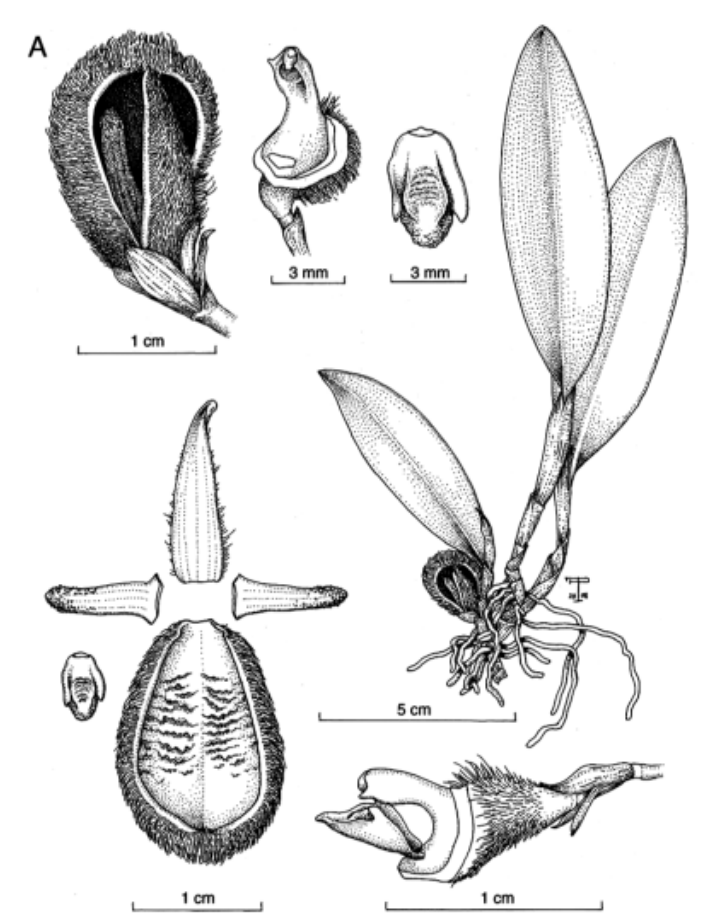

B

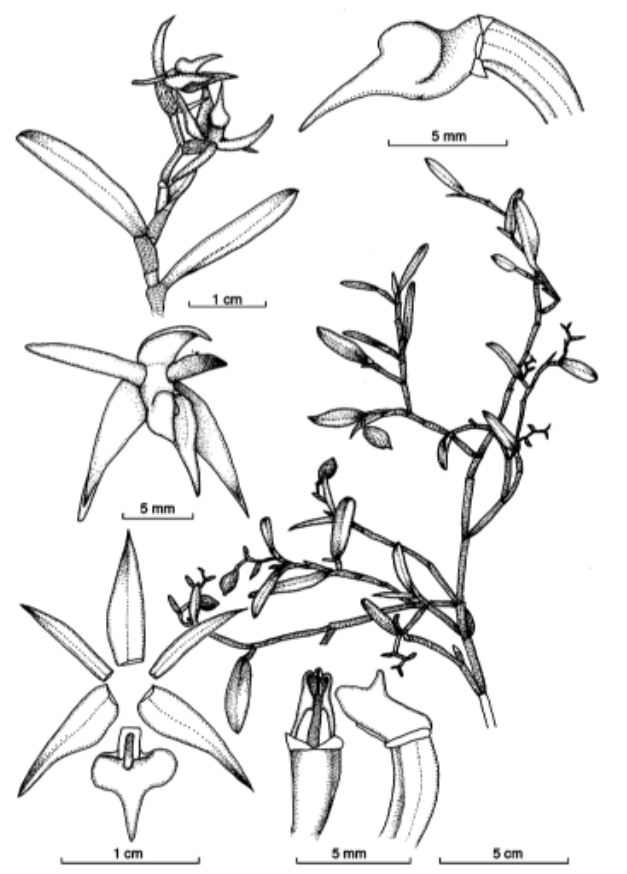

C
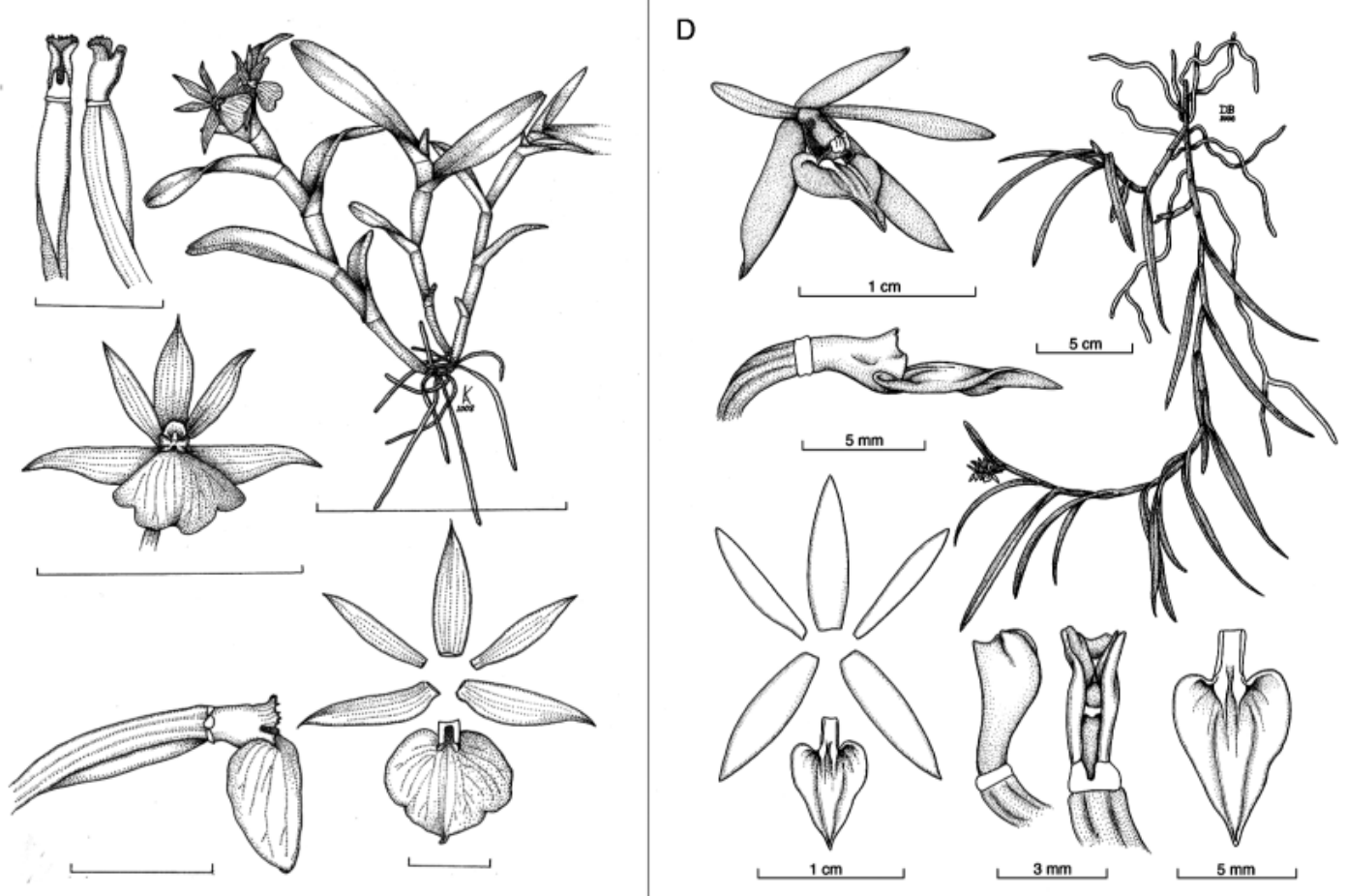

FiguRE 5. Drawings of: A. Echinosepala lappiformis; B. Epidendrum orthodontum; C. Epidendrum stellidifforme; D. Epidendrum zunigae. Drawings: A by Franco Pupulin, B, C by Adam Karremans, D by Diego Bogarín. 
Subtribe Maxillariinae: The genus Lycaste underwent several changes and additions after studies by Bogarín (2007) and Oakeley (2008). A new species restricted to the Pacific watershed of the Talamanca range was described as Lycaste bruncana Bogarín (Fig. 2h). It is closely allied to L. tricolor Rchb.f. but differs mainly in the twisted column and the shape of the callus and pollinarium. Geographic isolation and molecular evidence also support the distinction between these two species. Lycaste bruncana is conspecific with the laterdescribed Lycaste crystallina Wubben ex Oakeley. Lycaste brevispatha (Klotzsch) Lindl. \& Paxton is regarded as a synonym of $L$. candida Lindl., and, according to Oakeley (2008), L. angelae Oakeley should replace L. brevispatha. However, the application of the names Lycaste brevispatha and Lycaste candida is controversial. Lindley (1851) cited Lycaste candida as a nomen nudum. Later, in 1851, J. F. Klotzsch published Maxillaria brevispatha, which Lindley and Paxton transferred to Lycaste in 1853. Then Reichenbach recognized the distinctiveness of $L$. candida and validated Lindley's name in 1863. Oakeley (2008) regarded L. brevispatha as a synonym of $L$. candida but referring to the name L. candida Lindl. nom. nud. and not to L. candida Lindl. ex Rchb.f. of 1863 . The latter is predated by $L$. brevispatha (Klotzsch) Lindl. \& Paxton. So, if the two taxa are conspecific, L. brevispatha has priority over L. candida. A careful analysis of the application of the names is needed to clarify the status of the species of this group. A natural hybrid Lycaste $\mathrm{x}$ daniloi Oakeley is also reported as a cross between $L$. angelae and L. candida. Lycaste angelae var. rubra Oakeley was proposed, but it was ostensibly typified by a photo, and therefore it should regarded as an illegitimate name according to the the International Code of Botanical Nomenclature (Art. 37.4) which specifies that the type must be a specimen after 1 January 2007. The nomenclature of the Lycaste macrophylla (Poepp. \& Endl.) Lindl. complex, formerly represented in Costa Rica by three subspecies (L. macrophylla subsp. desboisiana (Cogn.) Fowlie, L. macrophylla subsp.
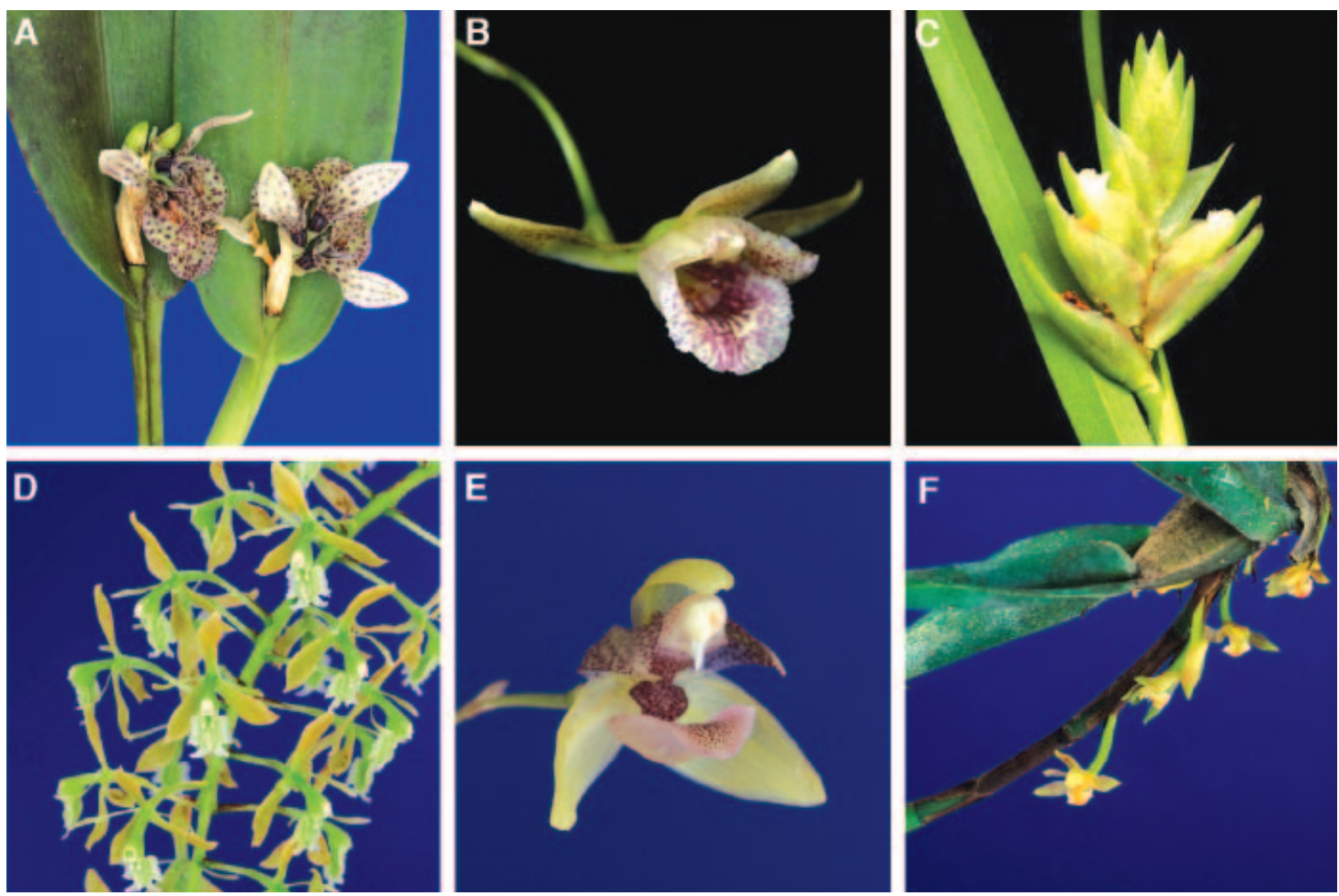

FIgURE 6. Photographs of: A. Acianthera hamata; B. x Bensteinia ramonensis; C. Elleanthus ligularis; D. Epidendrum isthmioides; E. Kefersteinia saccata; F. Ornithidium pendulum. Photographs: A, D, K, E, F by Diego Bogarín; B, courtesy of LBG. 
puntarenasensis Fowlie, and L. macrophylla subsp. xanthocheila Fowlie), also changed in Oakeley (2008). He segregated the taxa within this complex, attributing to Costa Rica the following species: L. xanthocheila (Fowlie) Oakeley, L. panamanensis Fowlie ex Oakeley (first record for Costa Rica), L. puntarenasensis (Fowlie) Oakeley, and L. measuresiana (B.S.Williams) Oakeley. Lycaste macrophylla subsp. desboisiana was treated as synonym of L. measuresiana (Oakeley, 2008). However, a previous publication by Archila (2002) seems to have priority over Oakeley (2008), so that the correct names are Lycaste puntarenasensis (Fowlie) Archila, Lycaste xanthocheila (Fowlie) Archila, and Lycaste panamanensis (Fowlie) Archila.

There are new additions to various genera segregated from Maxillaria s.l. (Blanco et al., 2007). Maxillaria bolivarensis C.Schweinf. described from Venezuela and distributed throughout South America has been recorded based on two collections along the Caribbean lowlands (Fig. 4I, 7b); the recent nomenclatural changes did not affect this name (Blanco 2008). Ornithidium pendulum (Poepp. \& Endl.) Cogn. (=Maxillaria pendula (Poepp. \& Endl.) C.Schweinf.), a widespread species ranging from Guatemala, Nicaragua, Panama, and South America, is recorded for a first time in Costa Rica (Fig. 6f, 7c). The misapplied name Maxillaria ramosa Ruiz \& Pav. has been widely used since its clarification by Blanco et al. (2008). Maxillaria appendiculoides C.Schweinf., first described from Costa Rica, has recently been reported from Ecuador (M. Blanco, personal communication). A new species of Mormolyca, allied to what we call the Maxillaria rufescens alliance, was described by Bogarín and Pupulin (2010) (Fig. 2i). Tribe Neottieae: Palmorchis nitida Dressler, previously described from Panama, was documented in the Golfo Dulce region of southern Costa Rica by Bainbridge and Aguilar (2008). Subtribe Oncidiinae: A collection of Lockhartia similar to L. micrantha Rchb.f. has been reported as the Colombian Lockhartia chocöensis Kränzl. based on two collections from the tropical wet forest-premontane belt transition on the Caribbean watershed of the Cordillera de Tilarán range northwestern Costa Rica (Fig. 4h, 7a). Studies in Trichopilia revealed a new species, $T$. primulina Dressler \& Bogarín, from a cultivated plant without specific locality (Dressler and Bogarín, 2009; Fig. 21, 11b). A recent collection is the first known locality of this species. The only record of Warmingia in Costa Rica, W. margaritacea B. Johans., is now considered a synonym of the Ecuadorian $W$. zamorana Dodson (Bogarín et al., 2007; Fig. 4k, 41, 11c, 11d).

Subtribe Pleurothallidinae: This is one of the largest groups represented in Costa Rica. After the significant contributions by Luer $(1987,2003)$ and studies by Pupulin (2002), Pupulin and Bogarín (2007), and Pupulin et al. (2007), seven new species and two new records have been registered. Three new species of Acianthera have been described: A. cabiriae Pupulin, G.A.Rojas \& J.D.Zúñiga (Fig. 8c), A. fecunda Pupulin, G.A.Rojas \& J.D.Zúñiga (Fig. 8b), both of these from the Caribbean lowlands of the Turrialba region, and A. hamata Pupulin \& G.A.Rojas, from an unknown locality (Pupulin et al., 2007; Fig. 8 d). A collection from southern Cartago corresponds to the first locality known for A. hamata. All the new species are endemic to Costa Rica (Fig. 6a). A new record, $A$. aberrans (Luer) Pupulin \& Bogarín has been found along the Caribbean lowlands in tropical rain forest of the Sarapiquí region (Fig. 4a, 8a). This species was described from Veraguas, Panama.

The Panamanian Barbosella orbicularis Luer, previously cited by Pupulin (2002) on the basis of a sterile voucher, was registered from tropical wet forest along the Caribbean watershed of the Cordillera Central (Fig. 3a, 4b). A specimen of Dracula maduroi Luer (previously described from Bocas del Toro, Panama) was documented in a recent expedition to Volcán Cacho Negro in the Braulio Carrillo National Park in the Cordillera Central (Fig. 2b). A specimen of Dryadella fuchsii Luer, described from Honduras, was cited for Costa Rica by Luer (2005) based on collections by P. C. Standley and J. Valerio around the Tilarán area in the northwestern Costa Rica. The species had not been listed for Costa Rica by Luer (2003) or Ossenbach et al. (2007). The Nicaraguan Echinosepala lappiformis (A.H.Heller \& L.O.Williams) Pridgeon \& M.W.Chase (=Brenesia lappiformis (A.H.Heller \& L.O.Williams)) Luer was recorded based on two collections from the Caribbean lowlands at $500 \mathrm{~m}$ of elevation (Fig. 4c, 5a). Another specimen was collected near Guápiles, Limón, and flowered recently in cultivation.

Studies on Lepanthes yielded many new records and species in last few years. Three species L. gratiosa Pupulin \& D.Jiménez (Fig. 1d), L. 

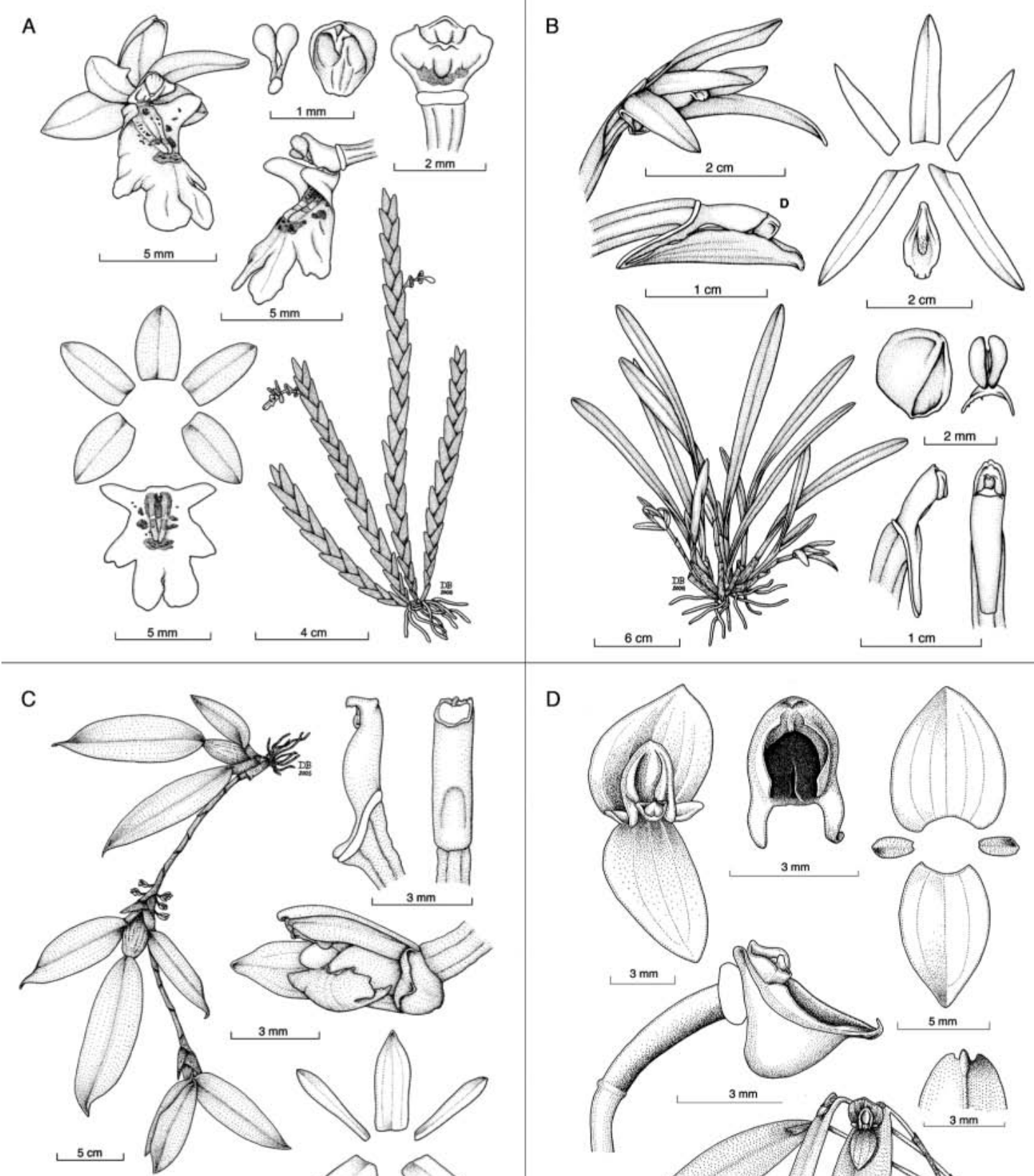

D

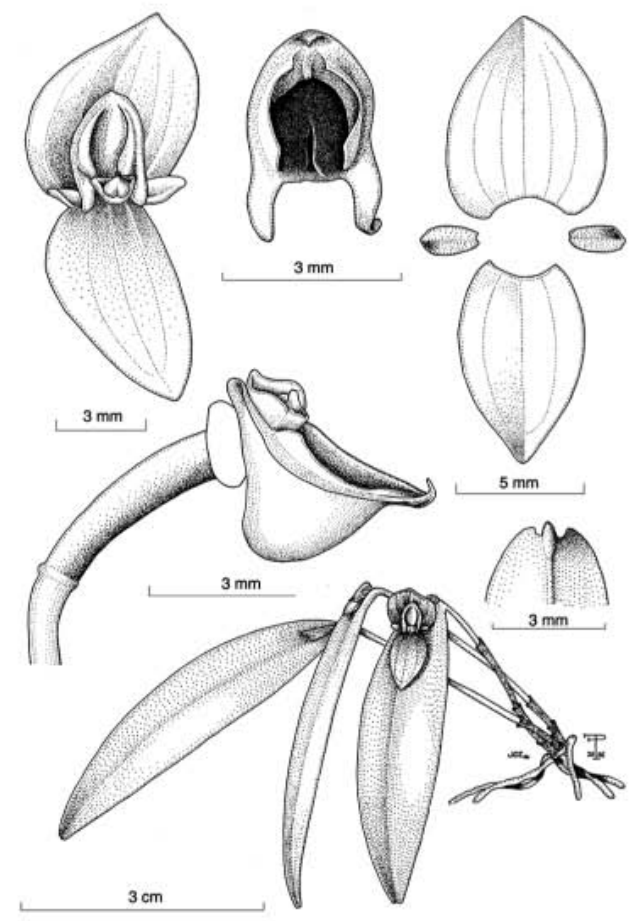

FIGURE 7. Drawings of: A. Lockhartia chocoensis; B. Maxillaria bolivarensis; C. Ornithidium pendulum; D. Pleurothallis bogarinii. Drawings: A, B, C by Diego Bogarín; D by Jose Daniel Zúñiga and Franco Pupulin. 


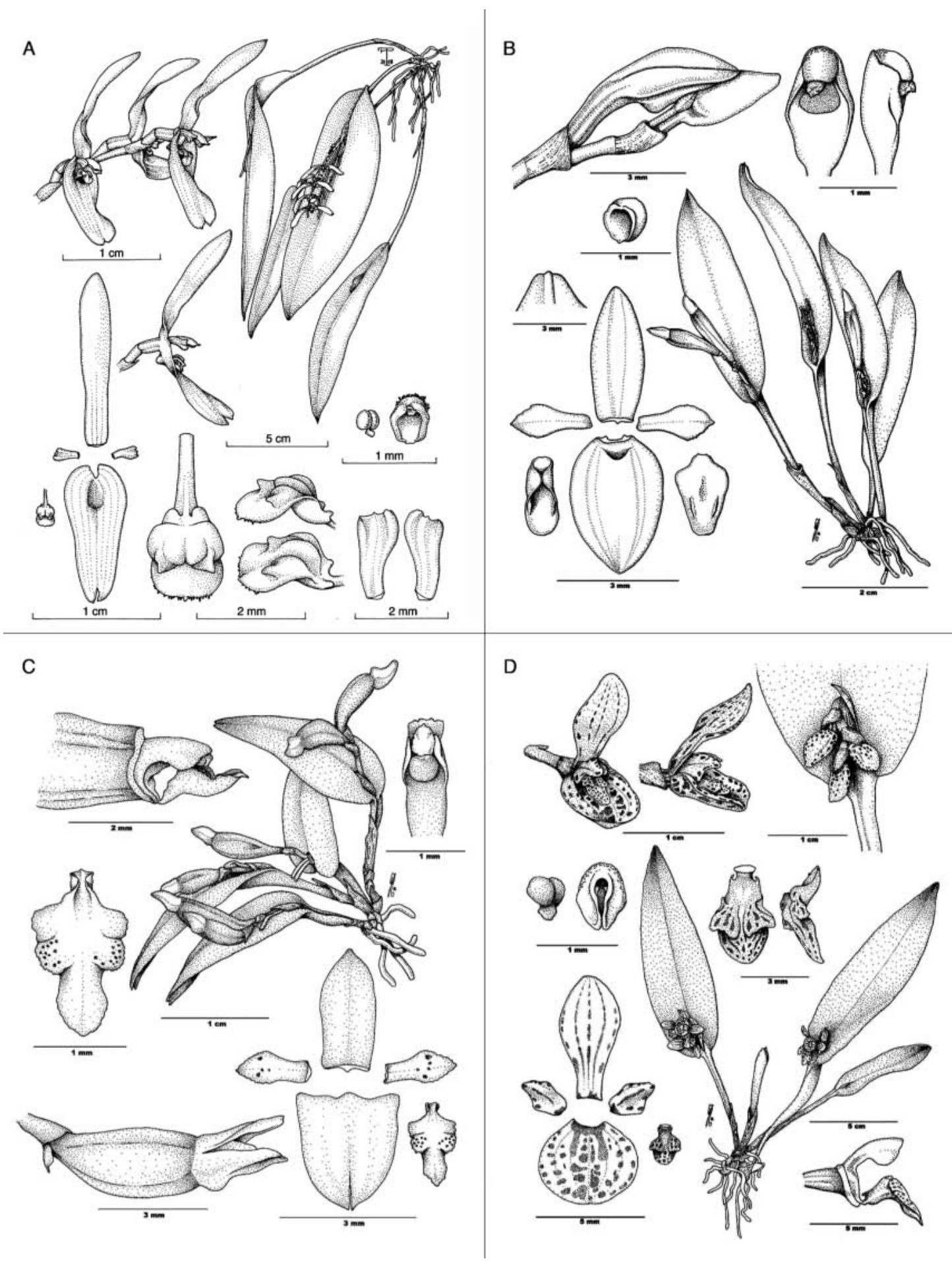

Figure 8. Drawings of: A. Acianthera aberrans; B. Acianthera cabiriae; C. Acianthera fecunda; D. Acianthera hamata. Drawings: A by Franco Pupulin; B, C, D by Franco Pupulin and Gustavo Rojas. 
A

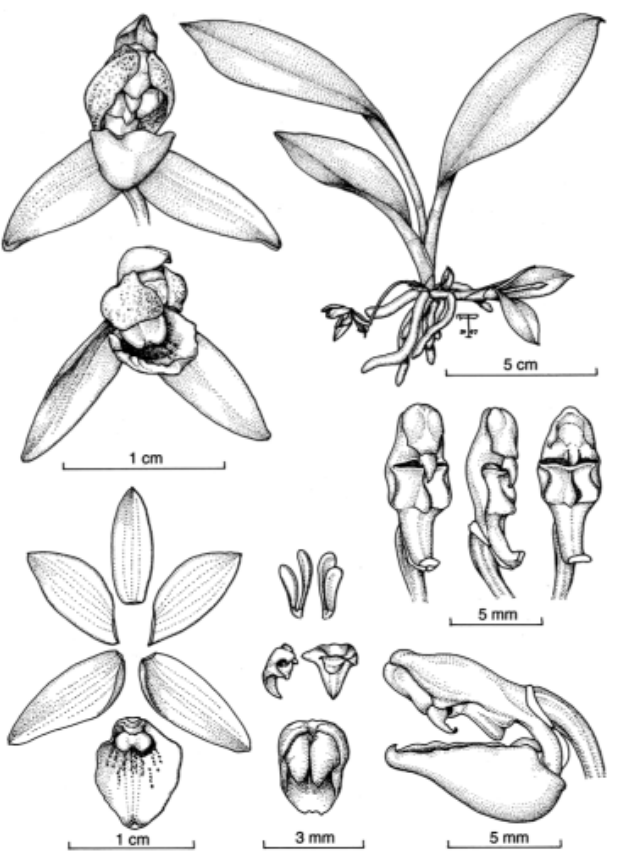

C

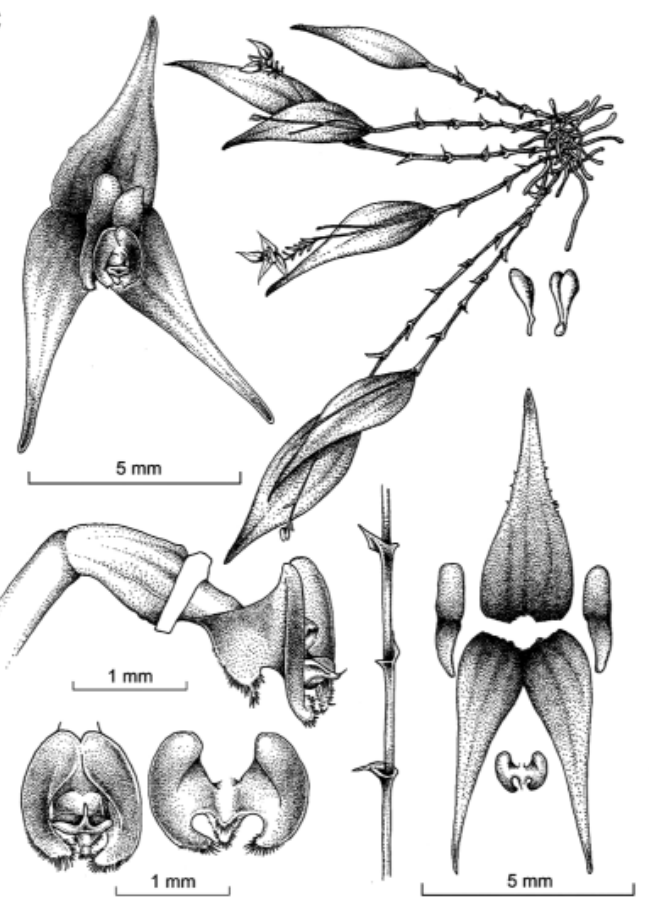

B
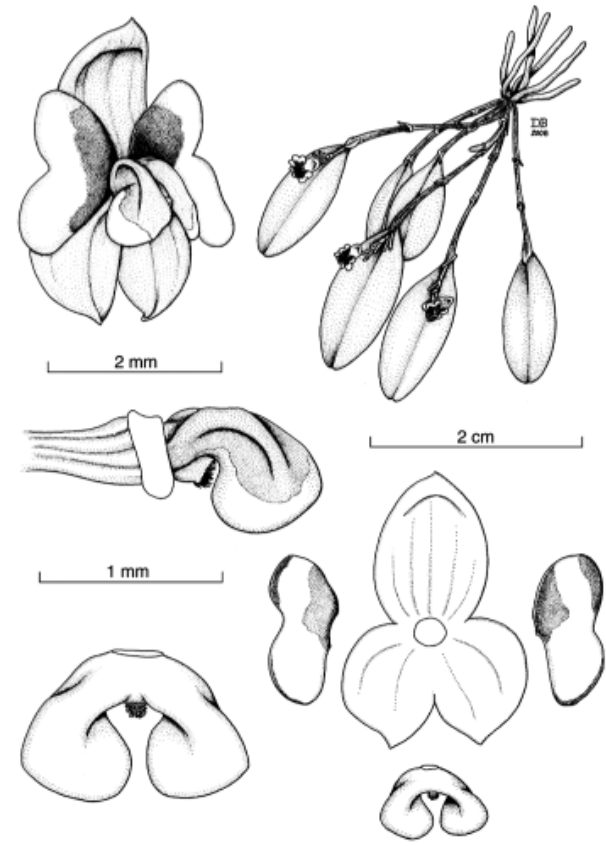

$1 \mathrm{~mm}$ $2 \mathrm{~mm}$
D

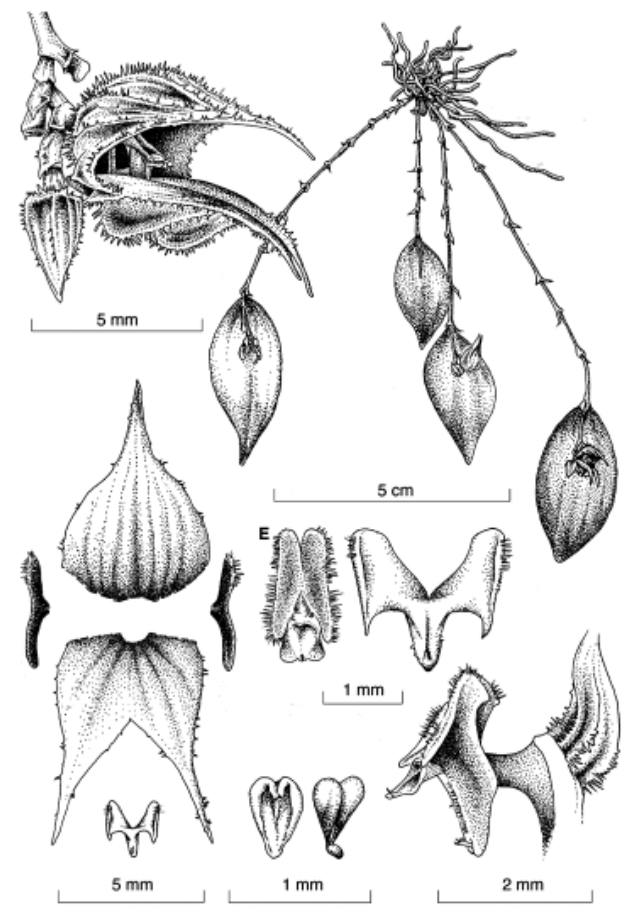

FIGURE 9. Drawings of: A. Kefersteinia saccata; B. Lepanthes mariposa; C. Lepanthes machogaffensis; D. Lepanthes pelvis. Drawings: A by Franco Pupulin; B by Diego Bogarín; C, D by Daniel Jiménez and Franco Pupulin.Pupulin. 


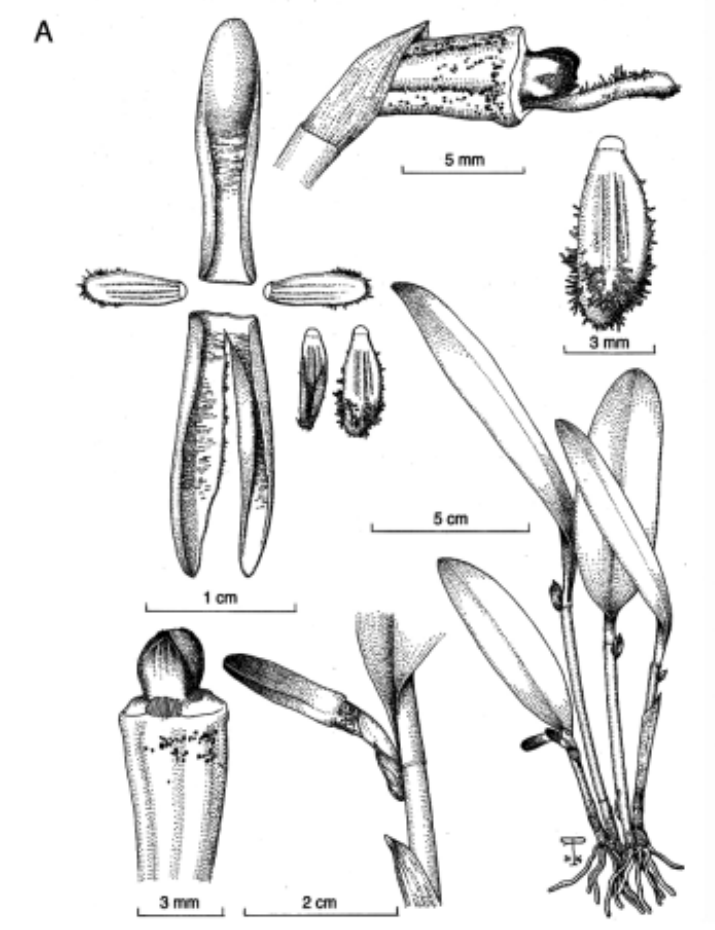

B
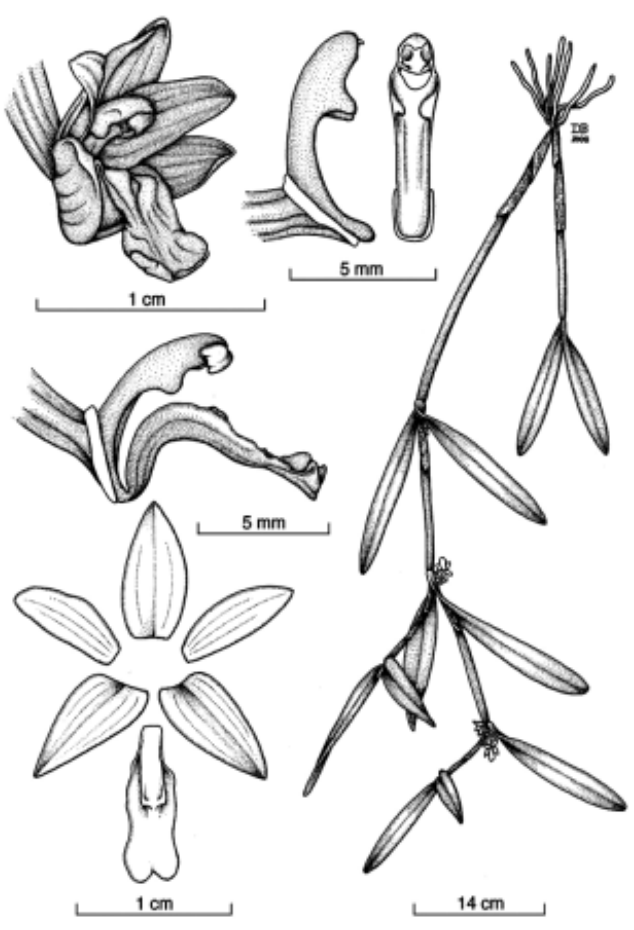

C

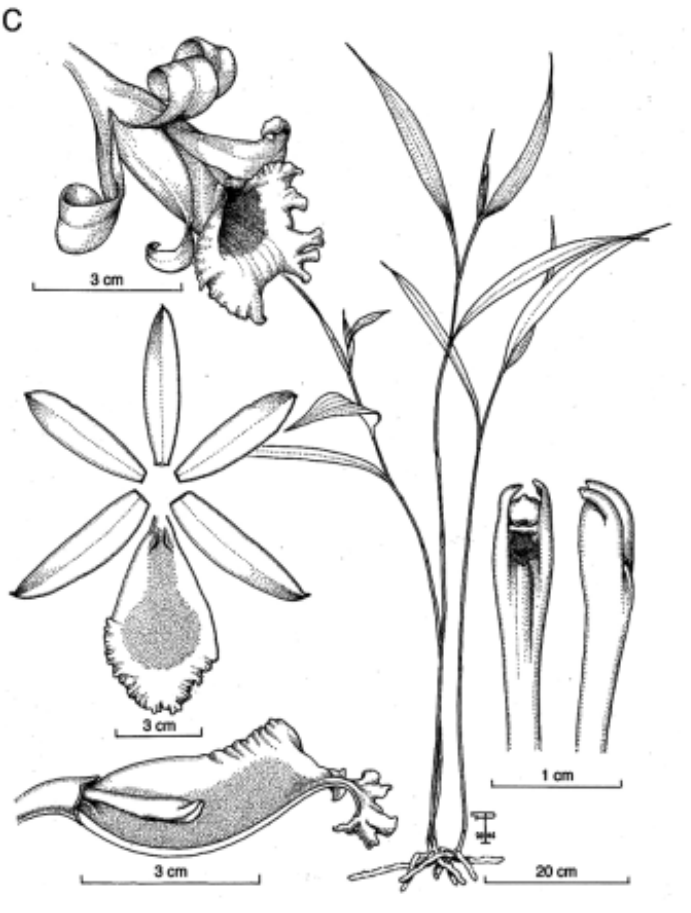

D

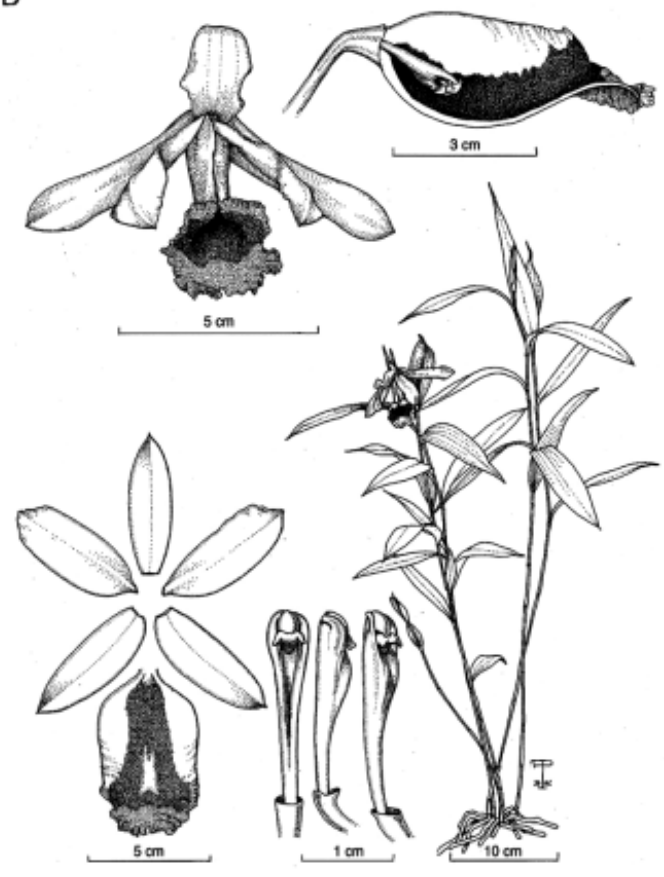

FIGURE 10. Drawings of: A. Restrepiella lueri; B. Scaphyglottis robusta; C. Sobralia aspera; D. Sobralia blancoi. Drawings: A, C, D by Franco Pupulin; B by Diego Bogarín. 


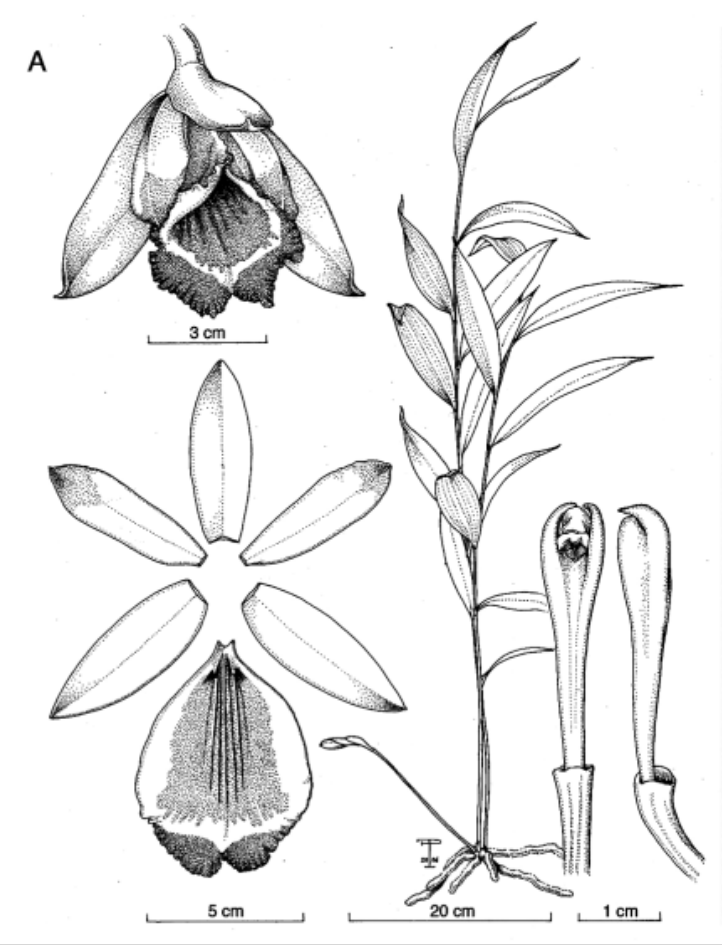

B
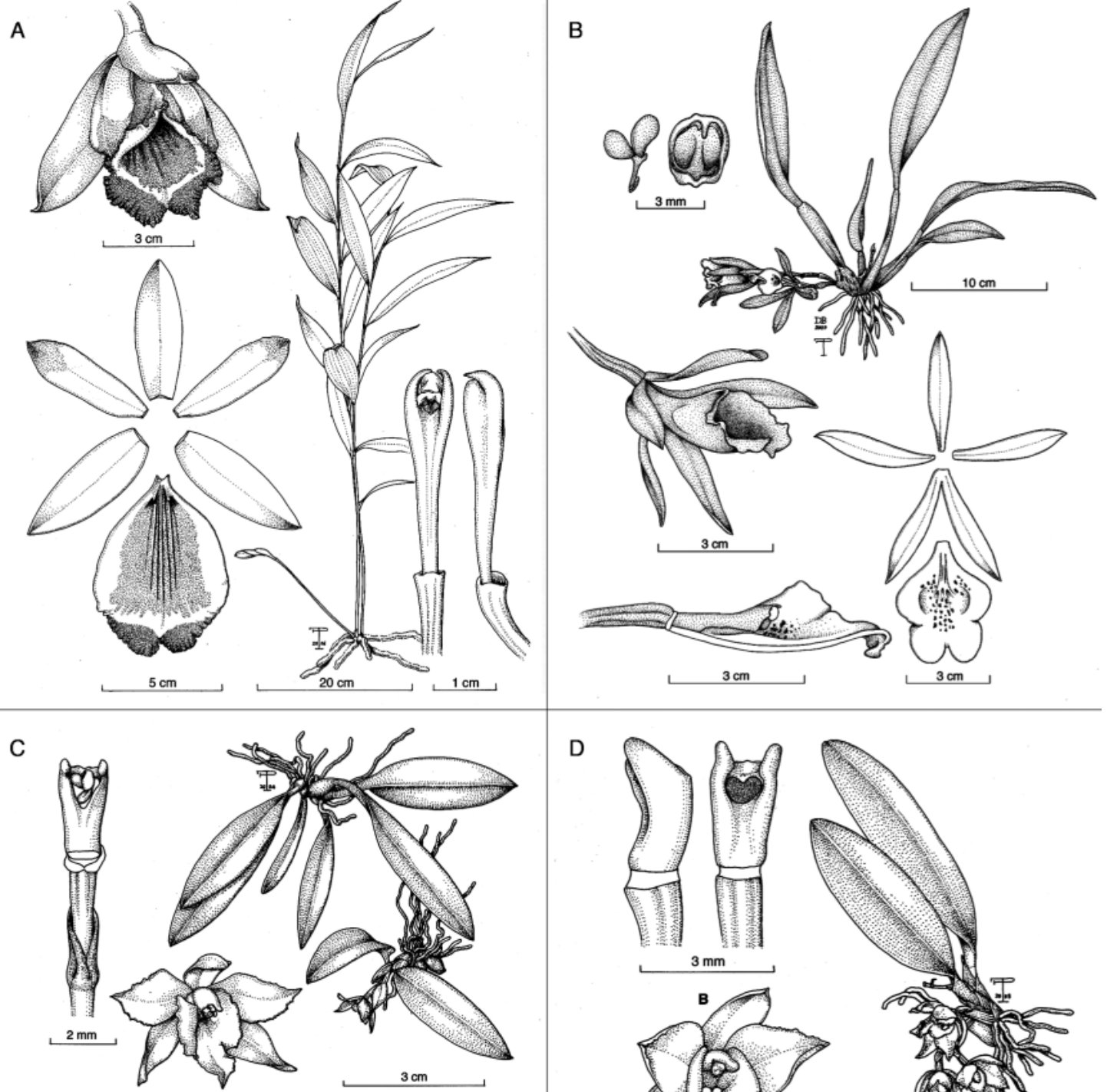

D
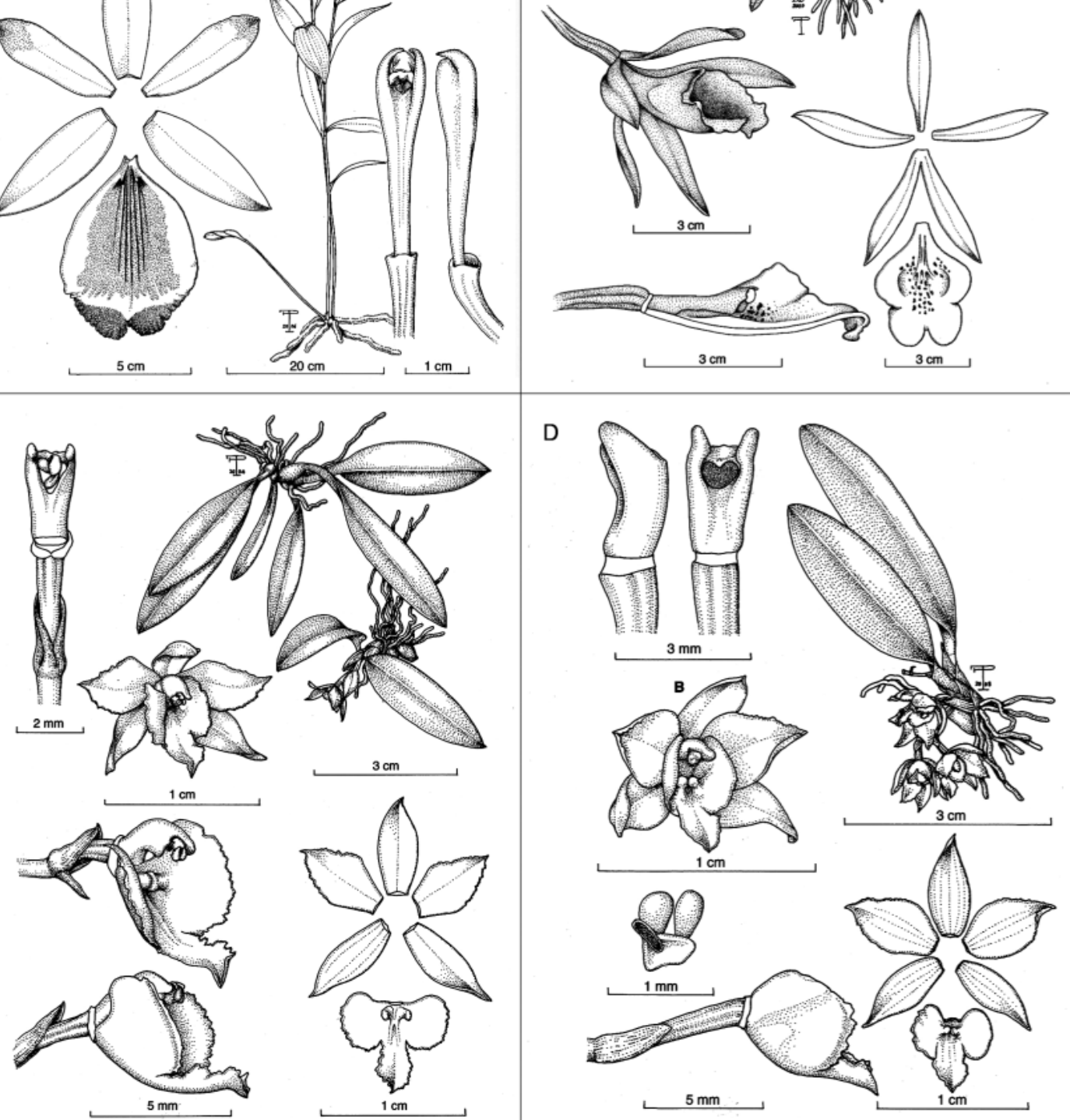

$\mathrm{D}$
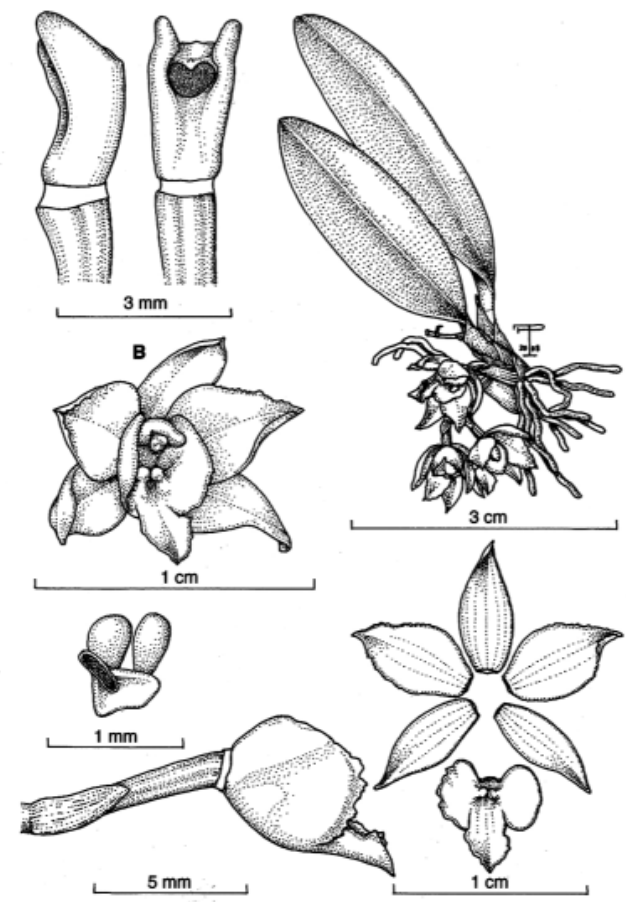

FigURE 11. Drawings of: A. Sobralia pendula; B. Trichopilia primulina; C. Warmingia zamorana (Costa Rica); D. Warmingia zamorana (Ecuador). Drawings: A, C, D by Franco Pupulin; B by Franco Pupulin and Diego Bogarín. 
machogaffensis Pupulin \& D.Jiménez (Fig. 2e, 9c), and L. pelvis Pupulin \& D.Jiménez (Fig. 2g, 9d) from the Cordillera de Talamanca - were recorded from a single path in Tapantí National Park. New collections apart from those already known have been registered for L. pelvis and L. machogaffensis. Also, L. droseroides Luer (Fig. 2c), L. mariposa Luer (Fig. 2f, 9b), and L. pexa Luer (of which it has not been possible to prepare a proper voucher from a cultivated plant collected in Central Volcanic range), all formerly endemic to Panama, have been collected in Costa Rica. Unpublished manuscripts will deal with more novelties in this genus (Bogarín, unpublished; Pupulin and Bogarín, unpublished).

Myoxanthus speciosus (Luer) Luer was cited by Pupulin (2002) and Luer (2003) based on a plant cultivated in Switzerland and collected in "Cordillera de Talamanca, Sierra Hills near Corazón de Jesús, alt. 2100 m" in Puntarenas Province. Luer (2003) stated that its origin is doubtful in all the collection details including the country. However, material collected near Cerro de La Muerte, along the Panamerican Highway and flowered in the living collections at the Lankester Botanical Garden confirms the presence of this species in Costa Rica. A new Pleurothallis, $P$. bogarinii Pupulin \& J.D.Zúñiga, allied to $P$. saphipetala Luer and restricted to the Caribbean lowlands, has been registered based on two collections (Fig. 2j, 7d).

The former monospecific genus Restrepiella has a second species: Restrepiella lueri Pupulin \& Bogarín (Fig. 2k, 10a). It differs from Restrepiella ophiocephala (Lindl.) Garay \& Dunst. in the lip without basal lobes, hirsute at apex and ciliate along the margins, twice as long as the column and as long as the petals, the column without a foot and the free lateral sepals.

Two additional records added by Luer (2006) are: Pleurothallis caudatipetala C.Schweinf. (=Specklinia caudatipetala (C.Schweinf.) Luer, =Panmorphia caudatipetala (C.Schweinf.) Luer), previously known from Peru and Ecuador and collected in Costa Rica around Cascajal, northern San José; and P. minutalis Lindl. (Panmorphia minutalis (Lind1.) Luer, = Specklinia minutalis (Lindl.) Luer, = Anathallis minutalis (Lindl.) Pridgeon \& M.W. Chase), known from Mexico and Guatemala, and based on a collection of A.R. Endrés (Endrés s.n., W). A specimen of
Pleurothallis duplooyi Luer \& Sayers (=Specklinia duplooyi (Luer \& Sayers) Luer, = Panmorphia duplooyi (Luer \& Sayers) Luer), formerly considered endemic to Belize, has been found in the Caribbean watershed of the Cordillera de Talamanca near Pejibaye in Cartago province. Plants are recognized by the shortly repent rhizome with prostrate lenticular leaves less than $8 \mathrm{~mm}$ long and purple flowers on a filiform peduncle. The specimen collected in Costa Rica shows an elliptic dorsal sepal rather than oblong, and basally wider lateral sepals; however, it is consistent with the drawing published by Luer (2006). The plant was sketched from a fertile specimen collected in the field, and one flower has been stored in the spirit collection at JBL. There is currently a project on the taxonomy and phylogeny of the Pleurothallidinae in Costa Rica being developed at the Lankester Botanical Garden. Without doubt many new additions will be revealed in the future.

Tribe Sobralieae: Ephemeral flowers, such as those of Sobralia, pose special problems for their identification, but a large living collection held at the Lankester Botanical Garden has allowed discovery of many new species. Sobralia aspera Dressler \& Pupulin (Fig. 9c), S. blancoi Dressler \& Pupulin (Fig. 9d), S. pendula Dressler \& Pupulin (Fig. 11a), and S. raraeavis Dressler were described as new, and a new record, the Panamanian S. bouchei Ames \& C.Schweinf, was found in Turrialba on the Caribbean lowlands. Three other new species of Sobralia are awaiting publication (Dressler and Bogarín, unpublished). In Elleanthus, there is a new species, E. ligularis Dressler \& Bogarín (Dressler and Bogarín, 2007; Fig. 6c). Plants were collected in Colombia, Costa Rica, Cuba, and Panama, but the species had remained unnamed in the absence of flowers. This species is similar to E. graminifolius (Barb.Rodr.) Løjtnant, but the plants are more robust with wider leaves. The blade of the lip is subquadrate rather than obtriangular, with a short base.

Subtribe Stanhopeinae: A new Gongora, Gongora boracayanensis Jenny, Dalström \& W.E.Higgins, was published from the Fila Costeña on the Pacific slope as result of a clarification of the misapplied use of the name Gongora quinquenervis Ruiz \& Pav. for a horticulturally known but scientifically undescribed Gongora from Costa Rica (Jenny et al., 2007). 
Subtribe Zygopetalinae: This subtribe has been well documented and revised in Costa Rica by Pupulin (2007). x Bensteinia ramonensis Pupulin, a natural bigeneric hybrid (Benzingia reichenbachiana (Schltr.) Dressler x Kefersteinia excentrica Dressler \& MoraRet.), was recorded in the Alberto Brenes Reserve on the Cordillera de Tilarán (Fig. 3b, 6b). In the revision of Dichaea of Costa Rica (Pupulin, 2007), a new species, Dichaea gomez-lauritoi Pupulin, was described from a specimen collected in Guápiles along the Caribbean plains of Limón (Fig. 3d). Four name changes are noted: Dichaea amparoana Schltr. (described from Costa Rica) is recognized as different from $D$. lankesteriAmes and removed from its synonymy. Dichaea standleyi Ames was placed as a synonym of D. acroblephrara Schltr. Dichaea schlechteri Folsom is now considered conspecific with $D$. similis Schltr. (formerly synonymized under $D$. cryptarrhena Rchb.f. ex Kränzl.). Dichaea ciliolata Rolfe was reduced to a synonym of $D$. hystricina Rchb.f. based on morphological observations (Pupulin, 2005). Two novelties appeared after the revision of Kefersteinia (Pupulin, 2001): Kefersteinia saccata Pupulin from the Caribbean lowlands (Pupulin and Merino, 2008; Fig. 6e, 9a), and a new record, the Panamanian $K$. alata, collected along Yorkín river on the Costa Rican side of the border with Panama (Pupulin, personal communication).

ACKNowledgements. I thank the scientific services of Costa Rican Ministry of Environment, Energy and Telecommunications (MINAET) and its National System of Conservation Areas (SINAC) for issuing the collecting permits under which wild species treated here were collected. I am also grateful to Robert L. Dressler, Adam Karremans, and Franco Pupulin for their continuous support and to Melania Fernández, Reynaldo Gómez, and Rafael Trejos for assisting during fieldwork activities and management of the collections at JBL. The present work is part of the Project 814-A7-015, "Inventario y taxonomía de la flora epífita de la región Neotropical - Orchidaceae", sponsored by the Vice-Presidency of Research, University of Costa Rica.

\section{LiTERATURE CITED}

Archila, F. 2002. Nuevas combinaciones en el género Lycaste (Orchidaceae). Rev. Guatemalensis 5: 58-62.

Bainbridge, C. V. \& R. Aguilar. 2008. A new addition to the Costa Rican flora: Palmorchis nitida (Orchidaceae:
Neottieae) is documented from the Osa Peninsula. Lankesteriana 8: 1-4.

Blanco, M. A., G. Carnevali, M. Whitten, R. B. Singer, S. Koehler, N. H. Williams, I. Ojeda, N. M. Neubig \& L. Endara. 2007. Generic realignments in Maxillariinae (Orchidaceae). Lankesteriana 7: 515-537.

Blanco, M. A., G. Carnevali, D. Bogarín \& R. B. Singer. 2008. Further disentangling of a taxonomic puzzle: Maxillaria ramosa, Ornithidium pendulum, and a new species, O. elianae (Orchidaceae). Harvard Pap. Bot. 13: 137-154.

Bogarín, D. 2007. A new Lycaste (Orchidaceae: Maxillarieae) from Costa Rica. Lankesteriana 7: 543-549.

Bogarín, D., A. Karremans \& F. Pupulin. 2008. New species and records of Orchidaceae from Costa Rica. Lankesteriana 8: 53-74.

Bogarín, D. and Pupulin, F. 2009. The genus Campylocentrum (Orchidaceae: Angraecinae) in Costa Rica: some critical questions and a few answers. Pp. 3245 in: A. M. Pridgeon and J. P. Suárez (eds.). Proceedings of the Second Scientific Conference on Andean Orchids. Universidad Técnica Particular de Loja, Loja, Ecuador.

Bogarín, D. \& F. Pupulin. 2010. Two new species of Mormolyca from Costa Rica and Panama. Orch. Digest 74: 43-47.

Dressler, R. L. 1993. Field guide to the orchids of Costa Rica and Panama. Cornell University Press, Ithaca, New York, USA.

Dressler, R. L. 2003. Orchidaceae. Pp. 1-595 in: B. E. Hammel, M. H. Grayum, C. Herrera \& N. Zamora (eds.). Manual de plantas de Costa Rica - Volumen III, monocotiledóneas (Orchidaceae-Zingiberaceae). Monogr. Syst. Bot. Missouri Bot. Gard. 93.

Dressler, R. L. \& D. Bogarín. 2007. Elleanthus ligularis, a name for a relatively common "new" species of Elleanthus sect. Chloidelyna. Lankesteriana 7: 539-542.

Dressler, R. L. \& D. Bogarín. 2009. Der Trichopilia tortilis komplex (Orchidaceae: Oncidiinae) mit einer schwierig $\mathrm{zu}$ bestimmenden neuen art. The Trichopilia tortilis complex (Orchidaceae: Oncidiinae) with an elusive new species. Orchideen J. 2: 56-65.

Hágsater, E., L. Sánchez-Saldaña \& J. García-Cruz. 2004. Icones Orchidacearum, Fasc. 7. The Genus Epidendrum. Part 4. Instituto Chinoin, A.C., México.

Hágsater, E., L. Sánchez-Saldaña \& J. García-Cruz. 2006. Icones Orchidacearum, Fasc. 8. The Genus Epidendrum. Part 5. Instituto Chinoin, A.C., México.

Hágsater, E. \& L. Sánchez-Saldaña. 2008. Icones Orchidacearum, Fasc. 11. The Genus Epidendrum. Part 7. Instituto Chinoin, A.C., México.

Hemsley, W. B. 1883. Orchideae. Pp. 197-308 in: F. D. Goldman \& O. Salvin (eds.). Biologia Centrali- 
Americana. R. H. Porter, London, UK.

Jenny, R., S. Dälstrom \& W. H. Higgins2007. An old species finally named and described: Gongora boracayanensis. Selbyana 28: 99-102.

Lindley, J. 1851. Lycaste leucantha. Paxton's Flower Gard. 2: 37 .

Lahaye, R., M. van der Bank, D. Bogarín, J. Warner, F. Pupulin, G. Gigot, O. Maurin, S. Duthoit, T. Barraclough \& V. Savolainen. 2008. DNA barcoding the floras of biodiversity hotspots. Proc. Nat. Ac. Sc. USA 105: $2923-$ 2928.

Luer, C. A. 1987. New Lepanthes species from Costa Rica and Panama. Lindleyana 2: 185-217.

Luer, C. A. 2003. Pleurothallis. Pp. 386-452 in: B. E. Hammel, M. H. Grayum, C. Herrera \& N. Zamora (eds.). Manual de plantas de Costa Rica - Volumen III, monocotiledóneas (Orchidaceae-Zingiberaceae). Monogr. Syst. Bot. Missouri Bot. Gard. 93.

Luer, C. A. 2005. Icones Pleurothallidinarum XXVII. Dryadella and Acronia section MacrophyllaeFasciculatae. Monogr. Syst. Bot. Missouri Bot. Gard. 103: 1-311.

Luer, C. A. 2006. Icones Pleurothallidinarum XXVIII. Reconsideration of Masdevallia, and the systematics of Specklinia and vegetatively similar genera (Orchidaceae). Monogr. Syst. Bot. Missouri Bot. Gard. 105: 21-244.

Mora-Retana, D. E. \& J. B. Castro. 1992. Lista actualizada de las orquídeas de Costa Rica (Orchidaceae). Brenesia 37: 79-124.

Oakeley, H. F. 2008. Lycaste, Ida and Anguloa: the essential guide. The author, Beckenham, Kent, UK.

Ossenbach, C., F. Pupulin \& R. L. Dressler. 2007. Orquídeas del istmo centroamericano: catálogo y estado de conservación/Checklist and conservation status: Orchids of the Central American isthmus. Editorial 25 de Mayo, Sabanilla de Montes de Oca, San José, Costa Rica.

Ormerod, P. 2007. Studies of Neotropical Goodyerinae (Orchidaceae). Harvard Pap. Bot. 9: 391-423.

Ormerod, P. 2008. Studies of Neotropical Goodyerinae (Orchidaceae). Harvard Pap. Bot. 13: 55-87.
Pupulin, F. 2001. Contributions to a reassessment of Costa Rican Zygopetalinae (Orchidaceae). The genus Kefersteinia Rchb.f. Ann. Naturhisto. Mus. Wien 103B: 525-555.

Pupulin, F. 2002. Catálogo revisado y anotado de las Orchidaceae de Costa Rica. Lankesteriana 4: 1-88.

Pupulin, F. 2005. Ciliate dichaeas; Dichaea hystricina and Dichaea ciliolata: two species in one and an interesting variation. Orchids 74: 678-683.

Pupulin, F. 2007. Contributions toward a reassessment of Costa Rican Zygopetalinae (Orchidaceae). 3. A systematic revision of Dichaea in Costa Rica. Harvard Pap. Bot. 12: 15-153.

Pupulin, F. \& D. Bogarín. 2004. Two new species of Lepanthes (Orchidaceae: Pleurothallidinae) from Costa Rica. Kew Bulletin 59: 559-563.

Pupulin, F. \& D. Bogarín. 2007. A second new species in the genus Restrepiella (Orchidaceae:Pleurothallidinae). Willdenowia 37: 323-329.

Pupulin, F., D. Bogarín \& D. Jiménez. 2009. New species and records in Mesoamerican Lepanthes. Orch. Digest 73: 136-145.

Pupulin, F. \& G. Merino. 2008. Two new species of Kefersteinia (Orchidaceae: Zygopetalinae). Willdenowia 38: 1-7.

Pupulin, F., G. Rojas \& J. D. Zúñiga. 2007. Three new species of Acianthera (Orchidaceae: Pleurothallidinae) from Costa Rica. Harvard Pap. Bot. 11: 155-162.

Reichenbach, H. G. 1866. Beiträge zu einer orchideenkunde Central-Amerika's. Hamburg, Germany.

Schlechter, R. 1918. Kritische aufzählung der bisher aus Zentral-Amerika bekanntgewordenen. Orchidaceen, Beihefte zum Botanischen Centralblatt. Verlag von C. Heinrich, Dresden, Germany.

Standley, P. C. 1937. Flora of Costa Rica (Part 1). Publications of Field Museum of Natural History, Botanical Series 18: 1-63.

Williams, L. O. 1956. An enumeration of the Orchidaceae of Central America, British Honduras and Panama. Ceiba 5: 1-2. 